\title{
Supporting Information: Designing
}

\section{Two-Dimensional Versatile Room-Temperature Ferromagnets via Assembling Large-Scale}

\section{Magnetic Quantum Dots}

Xiaofeng Liu, Xinming Qin, Xiangyang Li, Zijing Ding, Xingxing Li, Wei Hu, ${ }^{*}$ and Jinlong Yang*

Hefei National Laboratory for Physical Sciences at Microscale, Department of Chemical Physics, and Synergetic Innovation Center of Quantum Information and Quantum Physics, University of Science and Technology of China, Hefei, Anhui 230026, China

E-mail: whuustc@ustc.edu.cn; jlyang@ustc.edu.cn 
Computational Methods. The electronic structure calculations based on Kohn-Sham density functional theory are carried out by using the Spanish Initiative for Electronic Simulations with Thousands of Atoms (SIESTA) software package. ${ }^{1}$ The electronic structure is described based on the generalized gradient approximation (GGA) of Perdew, Burke, and Ernzerhof (PBE) exchange-correlation functional with collinear spin polarization, ${ }^{2}$ and the double zeta plus polarization orbital basis set (DZP) within the framework of a linear combination of numerical atomic orbitals (LCAO). ${ }^{3}$ The screened hybrid HSE06 functional ${ }^{4,5}$ implemented in HONPAS (Hefei Order-N Packages for ab initio Simulations based on SIESTA) ${ }^{6}$ is used to give more reliable electronic and magnetic properties. ${ }^{7-9}$ The structures are fully relaxed by using the conjugate gradient (CG) algorithm until the energy and force converge to $10^{-4} \mathrm{eV}$ and $0.02 \mathrm{eV} / \AA$, respectively. The thermostability of these proposed COFs and MOFs is checked by conducting AIMD simulations. The AIMD simulations are performed for 8 ps with a time step of 1.0 fs at 300 and $400 \mathrm{~K}$ controlled by a Nosé-Hoover thermostat. Before conducting the DFT calculations in SIESTA, we have cross-checked the electronic and magnetic properties of TZGDs and other graphene dots ${ }^{10,11}$ by utilizing the Vienna Ab-initio Simulation Package (VASP) software and make sure that the obtained results are reliable. ${ }^{12,13}$ To investigate the influence of the substrate, the DFT-D3 method proposed by Grimme et al. in VASP is used to account for the effect of the van der Waals (vdW) interaction. ${ }^{14}$

The band centers $\left(\varepsilon_{\sigma}\right)$ of the spin majority and minority are calculated by the formula: ${ }^{15,16}$

$$
\varepsilon_{\sigma}=\frac{\int_{-\infty}^{+\infty} E D_{\sigma}\left(E-E_{F}\right) d \varepsilon}{\int_{-\infty}^{+\infty} D_{\sigma}\left(E-E_{F}\right) d \varepsilon}
$$

where $D_{\sigma}\left(E-E_{F}\right)$ is the projected density of states (PDOS) on the partial $\mathrm{p}(\mathrm{d})$-orbitals of the $\mathrm{C}, \mathrm{Si}$, and $\mathrm{Cr}$ atoms for the majority and minority spin $(\sigma)$, and $E_{F}$ is the Fermi energy. In our evaluation of the band centers, the lower limit of the integral is determined by the lowest occupied energy level of PDOS, and the corresponding upper limit is kept at the same 
level with a value of $10 \mathrm{eV}$ above the Fermi level for comparison, which includes unoccupied states. It should be noted the band center is employed to understand the magnetic exchange interaction strength qualitatively.

Electronic structures of magnetic quantum dots: TZGDs. We investigate the electronic structures of TZGDs monomers, as shown in Figure S1. In the nearest-neighbor tight-binding (TB) model with zero on-site energy, TZGD_n possesses $n-1$ degenerate non-bonding zero-energy states (NBSs). ${ }^{17}$ When the nonzero on-site energy is introduced in the mean-field Hubbard model, the degenerate NBSs are split up to the singly occupied and unoccupied molecular orbitals (MOs), which have opposite spin alignment, resulting in a Coulomb gap. ${ }^{17-19}$ The calculated ground states of TZGDs monomers are FM, where the magnetic moments are mainly localized on the zigzag edge $\mathrm{C}$ atoms with an FM alignment and the zigzag edge $\mathrm{C}$ atoms take the opposite spin-polarization direction compared with that of the minority sublattice $\mathrm{C}$ atoms. The calculated spin $(S)$ of TZGD_n monomers is $S=$ $(n-1) / 2$ in good agreement with Lieb's theorem. ${ }^{20,21}$ With the increase in the monomer's size, the total magnetic moment increases. Meanwhile, the gap, defined by the energy difference between the highest occupied MO and the lowest unoccupied MO of TZGD_n monomers, also decreases gradually and approaches zero, which agrees with previous reports well. ${ }^{17,22}$ The calculated gaps of TZGDs monomers with the PBE functional are underestimated below $1.0 \mathrm{eV}$. Through the hybrid functional (HSE06), the gaps of TZGDs are further revised. For instance, the calculated gap of TZGD_5 under the PBE functional is $0.76 \mathrm{eV}$, while it is 1.73 $\mathrm{eV}$ under the HSE06 functional and close to the experimental value $(1.70 \mathrm{eV}) .{ }^{23}$

Interestingly, TZGDs exhibit shocking potential in realizing a circular dichroism Hall effect and triplet excitonic insulator. ${ }^{24,25}$ In particular, large magnetic exchange coupling is determined in carbon-based materials, such as Clar goblet molecules $(23 \mathrm{meV}),{ }^{26}$ covalently linked nanographene dimers $(29 \mathrm{meV}),{ }^{27}$ thin $2 \mathrm{D}$ carbon nitride sheets $(59 \mathrm{meV}),{ }^{28}$ and rhombus-shaped nanographenes with zigzag periphery $(110 \mathrm{meV}),{ }^{29}$ which exceeds the thermal energy $\left(k_{\mathrm{B}} \mathrm{T}=26 \mathrm{meV}\right)$ and the Landauer limit $\left(k_{\mathrm{B}} \mathrm{Tln} 2=18 \mathrm{meV}\right)$ at room temperature 
and oversteps that of most reported inorganic magnetic semiconductors (a few meV). Combining with our calculations of ground-state properties, we expect that TZGDs have a more fascinating prospect in exploring room-temperature magnetism in 2D organic spintronics. In addition to magnetic quantum dot, FM order is also determined between the individual cluster magnetic moments in superatomic solids at low temperature (a few K) constructed by magnetic clusters and superatom $\left(\mathrm{C}_{60}\right) \cdot{ }^{30}$ It also gives us a hint to explore potential $2 \mathrm{D}$ ferromagnets by assembling molecular magnet building blocks with linkages into solids.

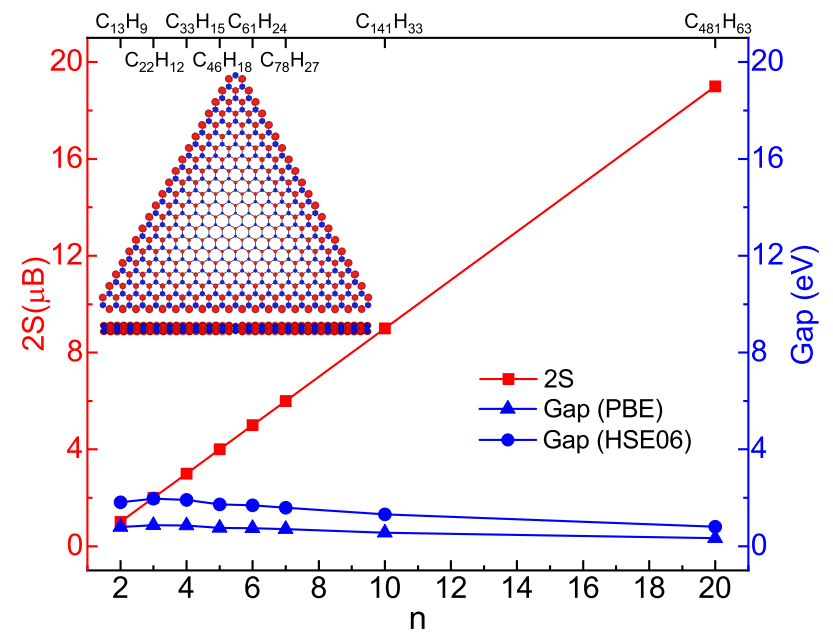

Figure S1 Scaling of spin moments $(2 S)$ and energy gaps with the size (n) of TZGD_ $n$ monomers under PBE and HSE06 functionals. The insert images show the top and side views of the spin density of TZGD_20 monomer with an isosurface value of $0.027 \mathrm{e} \cdot \AA^{-3}$ based on the HSE06 functional. Red and blue regions denote the spin majority and minority. The hydrogen atoms are omitted.

Stability of TZGDs. The thermostability of large-scale TZGDs is investigated, by conducting AIMD simulations for 4 ps with a time step of 1.0 fs at 300 and $400 \mathrm{~K}$, as shown in Figure $\mathrm{S} 2$ and $\mathrm{S} 3$. For example, the total energies of TZGD_10 $\left(\mathrm{C}_{141} \mathrm{H}_{33}\right)$ converge quickly within 0.5 ps (Figure S2). Though the corresponding planar geometric structures fluctuate slightly, the TZGD_10 can still maintain its basic frameworks. For the sake of computational cost, we also investigate the thermostability of TZGD_20 $\left(\mathrm{C}_{481} \mathrm{H}_{63}\right)$ monomer without spin polarization. Clearly, TZGD_20 can also maintain its basic framework expect slight fluctuation (Figure S3). Additionally, TZGD_6 monomer has been proved to keep its geometric structures with a critical temperature of $4200 \mathrm{~K} .{ }^{31}$ Thus, these TZGDs can stay 
stable against thermal fluctuation at room temperature.

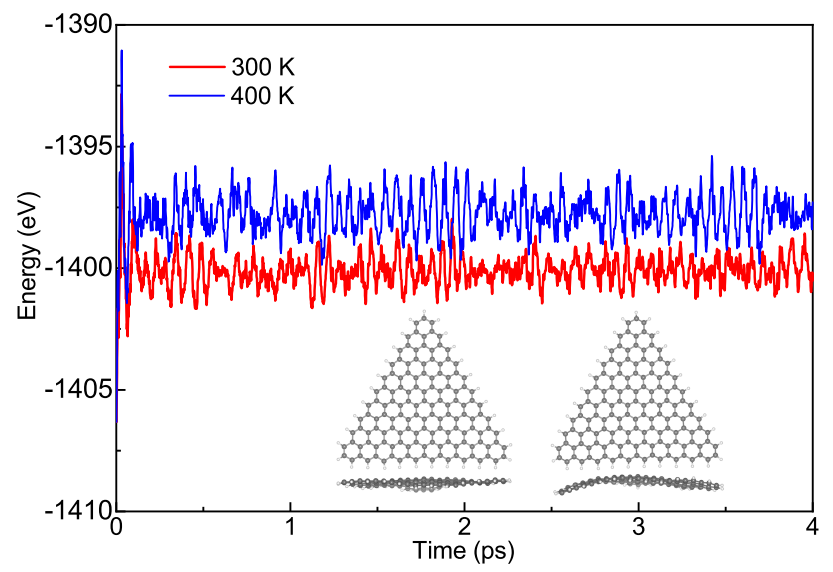

Figure S2 Energy fluctuations of TZGD_10 monomer as a function of time step by performing the AIMD simulation within 4 ps at 300 (red) and $400 \mathrm{~K}$ (blue). The inset graphs denote the top and side views of the optimized structure at the end of molecule dynamics process at 300 (left) and $400 \mathrm{~K}$ (right).

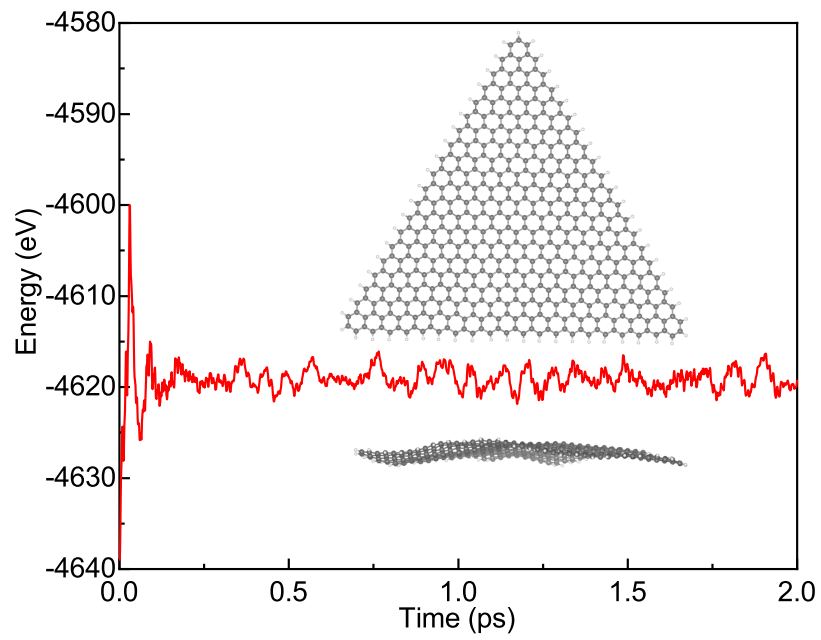

Figure S3 Energy fluctuations of TZGD_20 monomer as a function of time step by performing the AIMD simulation within 2 ps at $300 \mathrm{~K}$. The inset graphs denote the top and side views of the optimized structure at the end of molecule dynamics process. 


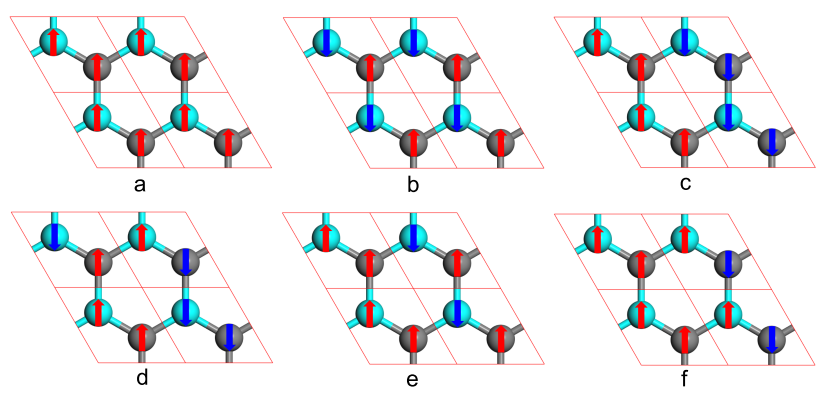

Figure S4 (a) Six magnetic configurations in a $2 \times 2 \times 1$ supercell for triangle lattices consisting of TZGDs with linkages. The blue and gray balls represent the linkages and TZGDs, respectively. (a) FF: TZGDs and linkages are in an FM arrangement, where the minority sublattice carbon atoms and linkages are in an AFM arrangement. (b) FFA: TZGDs and linkages are in an AFM arrangement. (c, d) AA1 and AA2: both TZGDs and linkages are in an AFM arrangement, respectively. (e) AF: TZGDs are in an FM arrangement and linkages are in an AFM arrangement. (f) FA: linkages are in an FM arrangement and TZGDs are in an AFM arrangement. Note: the magnetic configuration between TZGDs and linkages are defined by the majority sublattice carbon atoms and linkages. Red and blue arrows denote the spin polarization with different direction.

Table S1 Energy difference $(\mathrm{eV})$ between different spin arrangements and FFA for TZGD_n_2 triangle lattices consisting of TZGD_n $(\mathrm{n}=3,4,5,6,10$, and 20) and TZGD_2 as linkage. The energy of FFA is set to zero.

\begin{tabular}{lcccccc}
\hline \hline TZGD_n_2 & AA1 & AA2 & AF & FA & FF & FFA \\
\hline $3\left(\mathrm{C}_{35} \mathrm{H}_{15}\right)$ & 0.137 & 0.206 & 0.208 & 0.207 & 0.418 & 0 \\
$4\left(\mathrm{C}_{46} \mathrm{H}_{18}\right)$ & 0.138 & 0.208 & 0.210 & 0.208 & 0.419 & 0 \\
$5\left(\mathrm{C}_{59} \mathrm{H}_{21}\right)$ & 0.139 & 0.209 & 0210 & 0.208 & 0.419 & 0 \\
$6\left(\mathrm{C}_{74} \mathrm{H}_{24}\right)$ & 0.134 & 0.202 & 0.204 & 0.202 & 0.408 & 0 \\
$10\left(\mathrm{C}_{154} \mathrm{H}_{36}\right)$ & 0.138 & 0.208 & 0.210 & 0.208 & 0.421 & 0 \\
$20\left(\mathrm{C}_{494} \mathrm{H}_{66}\right)$ & 0.137 & 0.206 & 0.209 & 0.206 & 0.418 & 0 \\
\hline \hline
\end{tabular}



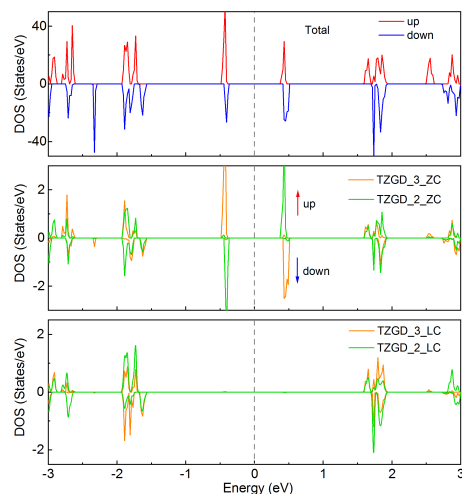

a
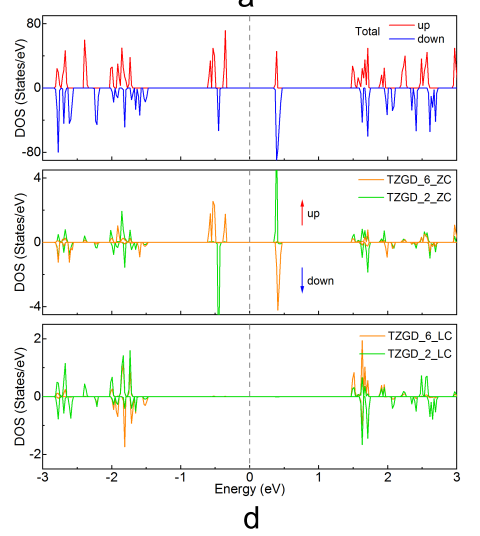
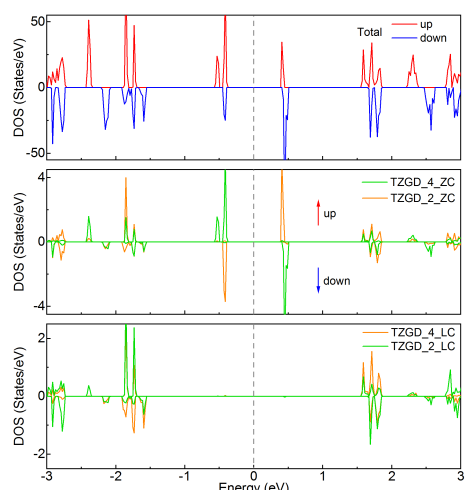

b
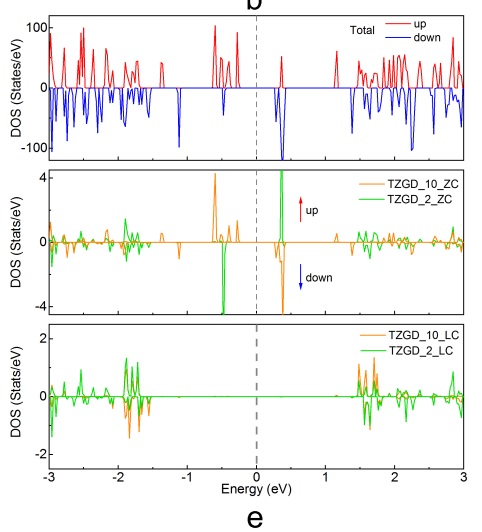
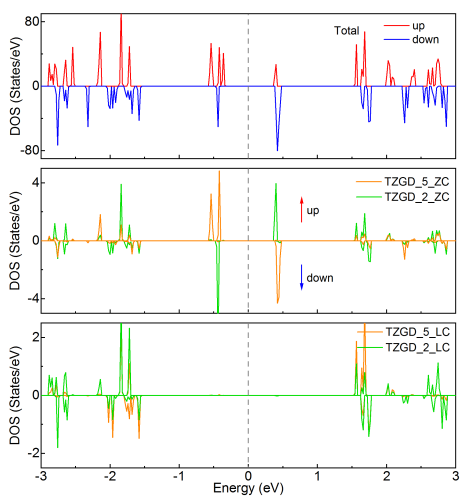

C
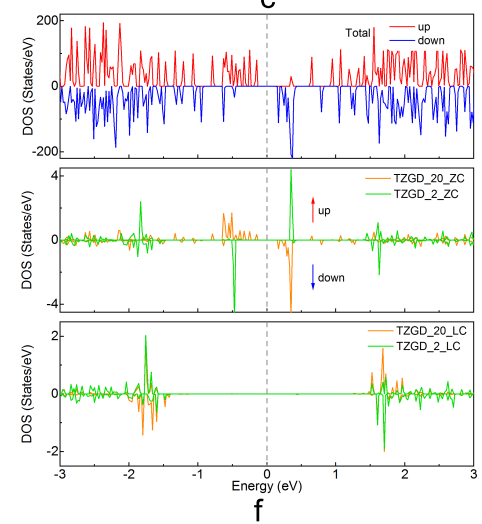

Figure S5 Total and projected density of states for the (a) TZGD_3_2, (b) TZGD_4_2, (c) TZGD_5_2, (d) TZGD_6_2, (e) TZGD_10_2, and (f) TZGD_20_2 triangle lattices based on TZGD_n and TZGD_2 under the PBE functional. TZGD_n_ZC and TZGD_2_ZC correspond to the zigzag edge carbon atoms. TZGD_n_LC and TZGD_2_LC correspond to the interconnected carbon atoms between TZGD_n and TZGD_2. 


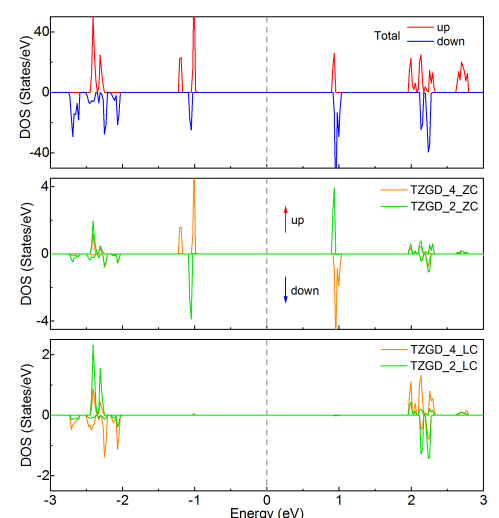

a

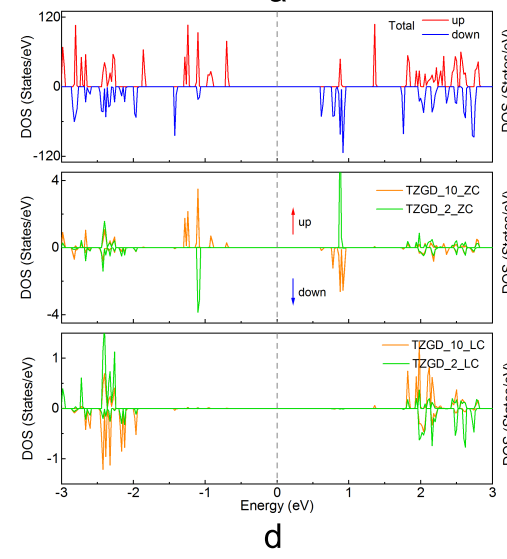

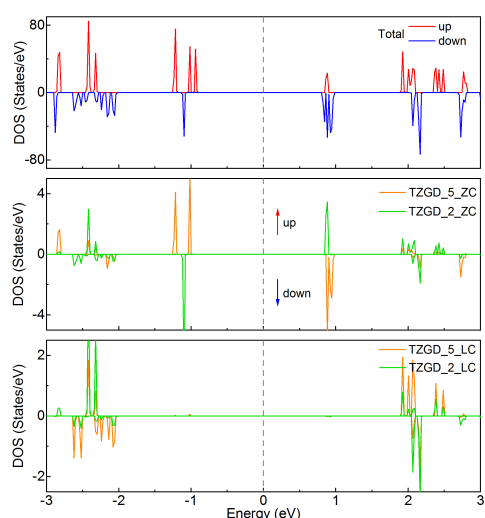

b

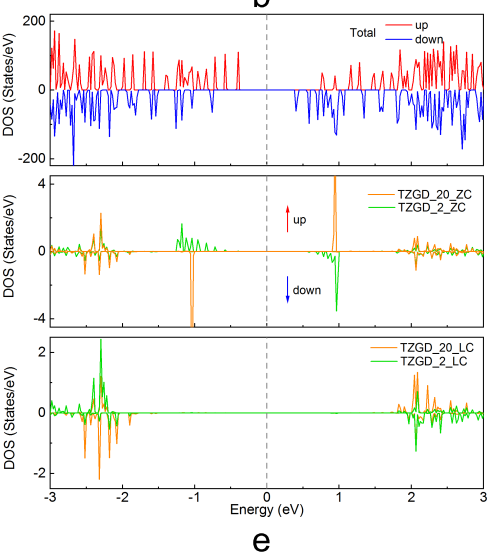

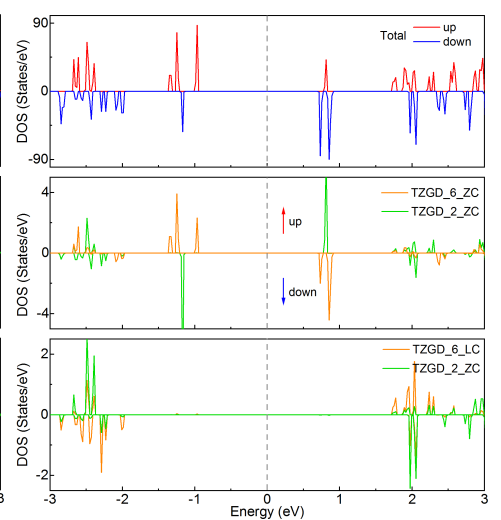

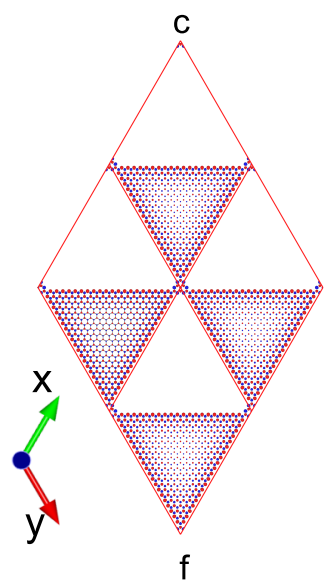

Figure S6 Total and projected density of states for the (a) TZGD_4_2, (b) TZGD_5_2, (c) TZGD_6_2, (d) TZGD_10_2, and (e) TZGD_20_2 triangle lattices based on the HSE06 functional. TZGD_n_ZC and TZGD_2_ZC correspond to the zigzag edge carbon atoms. TZGD_n_LC and TZGD_2_LC correspond to the interconnected carbon atoms between TZGD_n and TZGD_2. (f) Spin density of the TZGD_20_2 triangle lattice with an isosurface value of $0.027 \mathrm{e} \cdot \AA^{-3}$. The hydrogen atoms are not shown. Red and blue regions denote the spin majority and minority. Red lattice denotes the unit cell. 

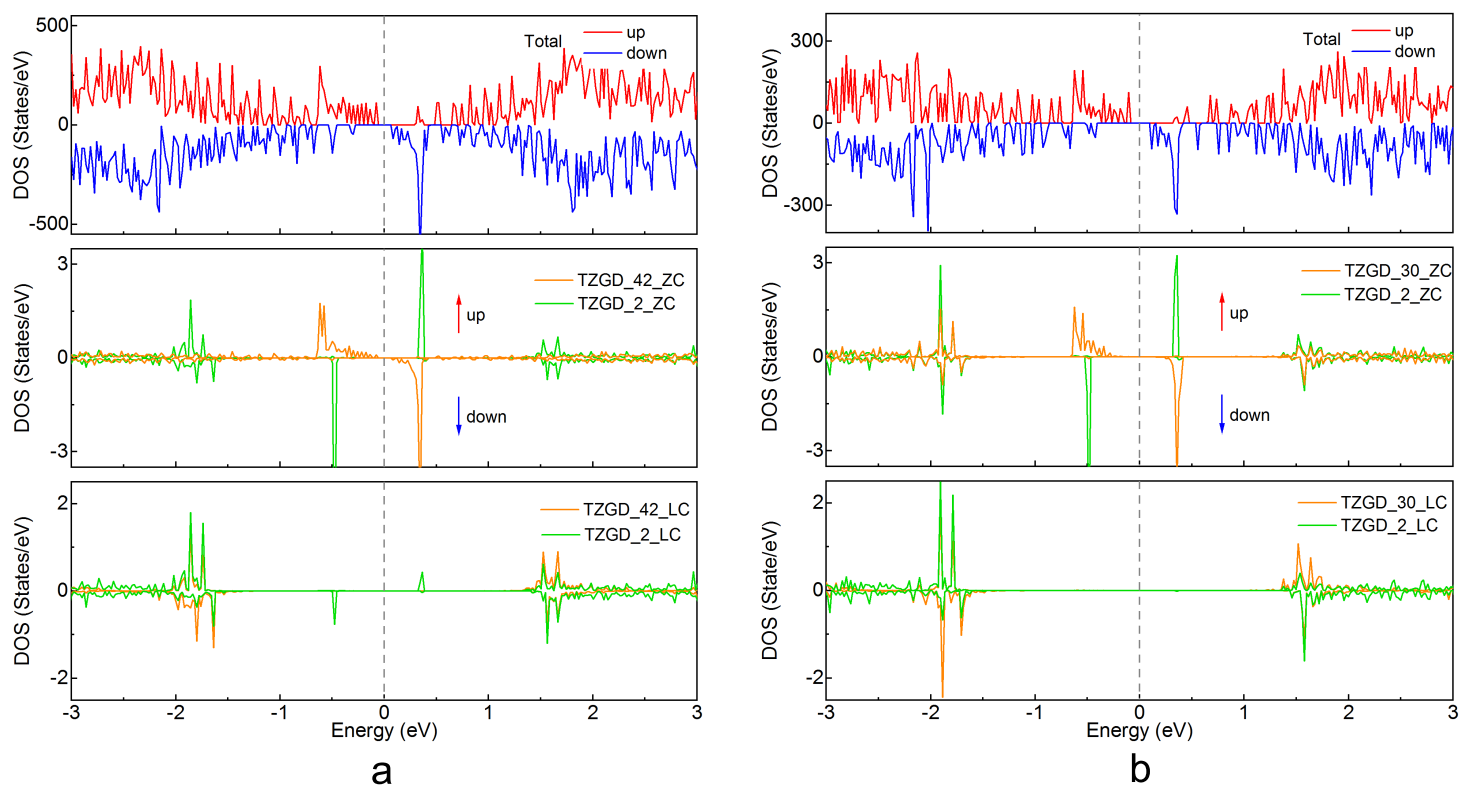

Figure S7 Total and projected density of states for the (a) TZGD_30_2 and (b) TZGD_42_2 triangle lattices based on the PBE functional. TZGD_n_ZC and TZGD_2_ZC correspond to the zigzag edge carbon atoms. TZGD_n_LC and TZGD_2_LC correspond to the interconnected carbon atoms between TZGD_n and TZGD_2. 


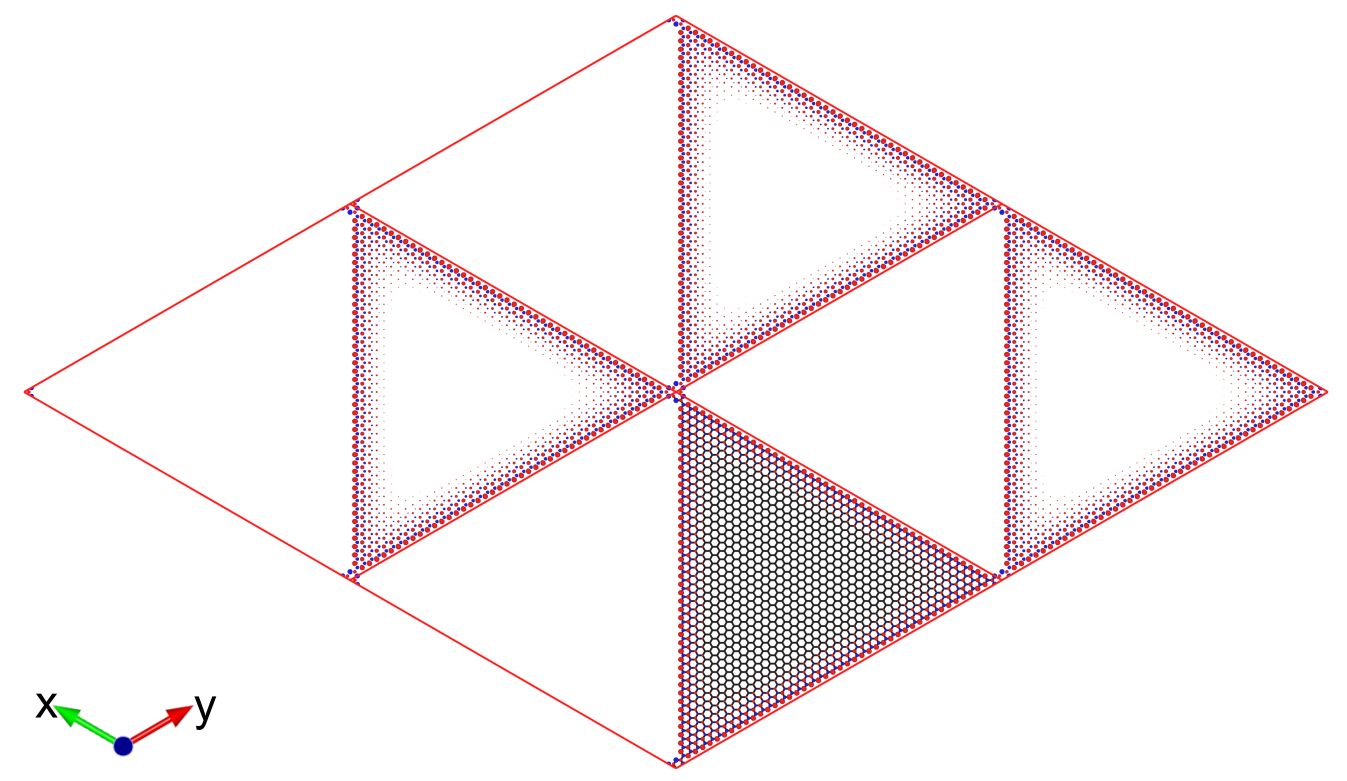

Figure S8 Spin density of the TZGD_42_2 triangle lattice with an isosurface value of $0.016 \mathrm{e} \cdot \AA^{-3}$. The hydrogen atoms are not shown. Red and blue regions denote the spin majority and minority. Red lattice denotes the unit cell. 
Table S2 Energy difference (eV) between different spin arrangements and FF for the TZGD_n_L triangle lattices with single atomic linkages $(\mathrm{Si}, \mathrm{V}, \mathrm{Cr}, \mathrm{Mn})$. The energy of $\mathrm{FF}$ is set to zero.

\begin{tabular}{cccccccc}
\hline \hline M & TZGD_N & AA1 & AA2 & AF & FA & FF & FFA \\
\hline Si & 2 & 0.770 & 0.583 & FF & 0.530 & 0 & FF \\
& 3 & 0.865 & 0.639 & FF & 0.629 & 0 & FF \\
& 4 & 0.855 & 0.631 & FF & 0.631 & 0 & FF \\
& 5 & 0.844 & 0.623 & FF & 0.624 & 0 & FF \\
& 6 & 0.826 & 0.610 & FF & 0.612 & 0 & FF \\
\hline Cr & 2 & 0.316 & 0.232 & 0.242 & 0.244 & 0 & 0.507 \\
& 3 & 0.395 & 0.293 & 0.305 & 0.293 & 0 & 0.610 \\
& 4 & 0.400 & 0.297 & 0.308 & 0.297 & 0 & 0.616 \\
& 5 & 0.393 & 0.292 & 0.303 & 0.292 & 0 & 0.606 \\
& 6 & 0.389 & 0.288 & 0.289 & 0.300 & 0 & 0.599 \\
\hline V & 2 & 0.308 & 0.239 & 0.224 & 0.222 & 0 & 0.407 \\
& 3 & 0.430 & 0.324 & 0.327 & 0.321 & 0 & 0.644 \\
& 4 & 0.425 & 0.316 & 0.323 & 0.317 & 0 & 0.648 \\
& 5 & 0.413 & 0.307 & 0.314 & 0.308 & 0 & 0.630 \\
& 6 & 0.411 & 0.305 & 0.306 & 0.312 & 0 & 0.627 \\
\hline Mn & 2 & 0.503 & 0.451 & 0.423 & 0.183 & 0 & 0.435 \\
& 3 & 0.414 & 0.331 & 0.325 & 0.235 & 0 & 0.460 \\
& 4 & 0.339 & 0.256 & 0.264 & 0.237 & 0 & 0.494 \\
& 5 & 0.299 & 0.222 & 0.232 & 0.220 & 0 & 0.463 \\
& 6 & 0.297 & 0.221 & 0.222 & 0.230 & 0 & 0.463 \\
\hline \hline
\end{tabular}



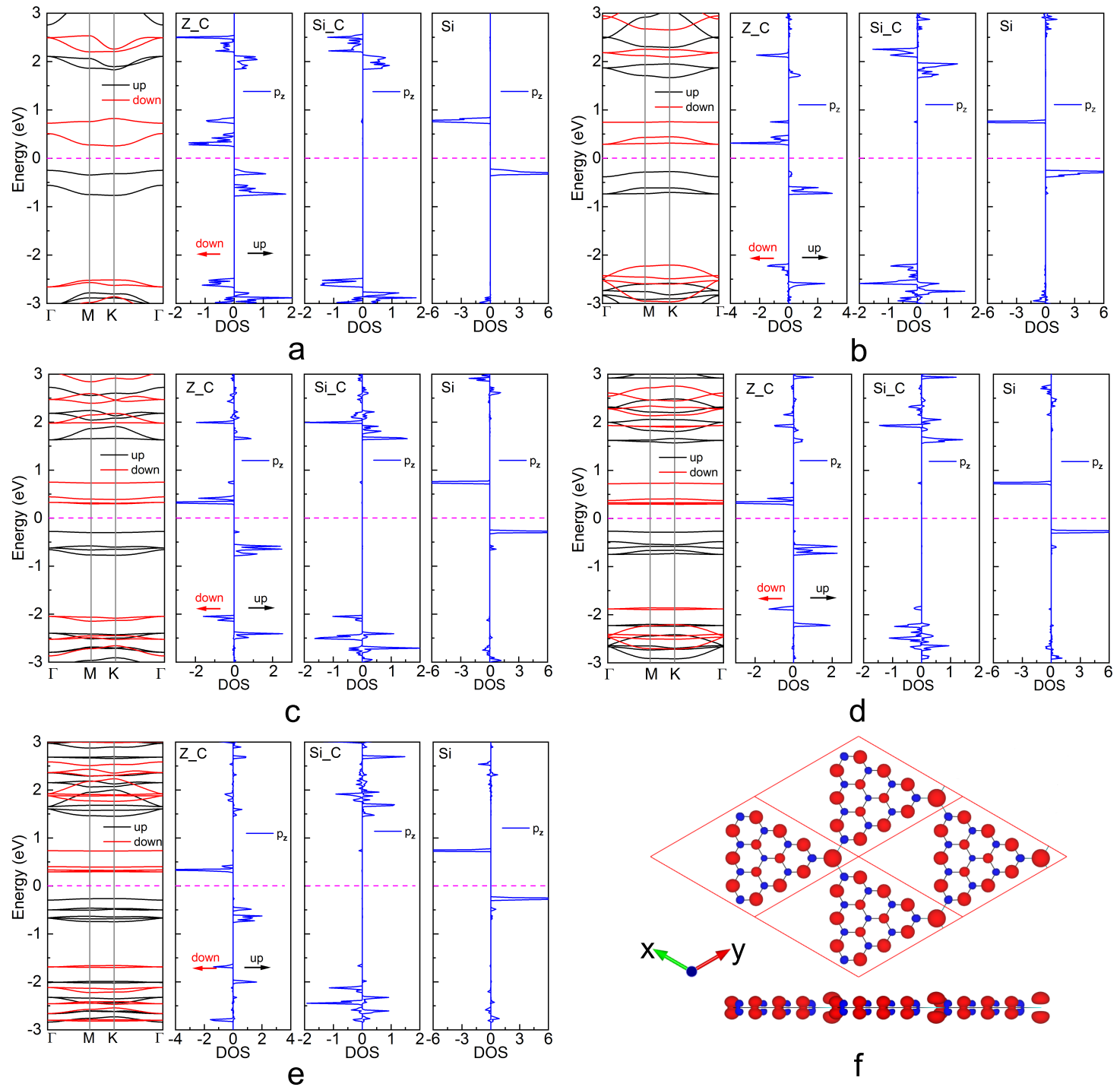

Figure S9 Band structures and projected density of states for the (a) TZGD_2_Si, (b) TZGD_3_Si, (c) TZGD_4_Si, (d) TZGD_5_Si, and (e) TZGD_6_Si triangle lattices. The Fermi energy is set to zero. The hydrogen atoms are omitted. $\mathrm{Z}_{-} \mathrm{C}, \mathrm{Si}-\mathrm{C}$, and $\mathrm{Si}$ denote the zigzag edge $\mathrm{C}$ atom, the interconnected $\mathrm{C}$ atom of TZGDs with $\mathrm{Si}$, and the $\mathrm{Si}$ atom. (f) Spin density of TZGD_3_Si with an isosurface value of $0.068 \mathrm{e} \cdot \AA^{-3}$. Red and blue regions represent the spin majority and spin minority. The hydrogen atoms are omitted. 

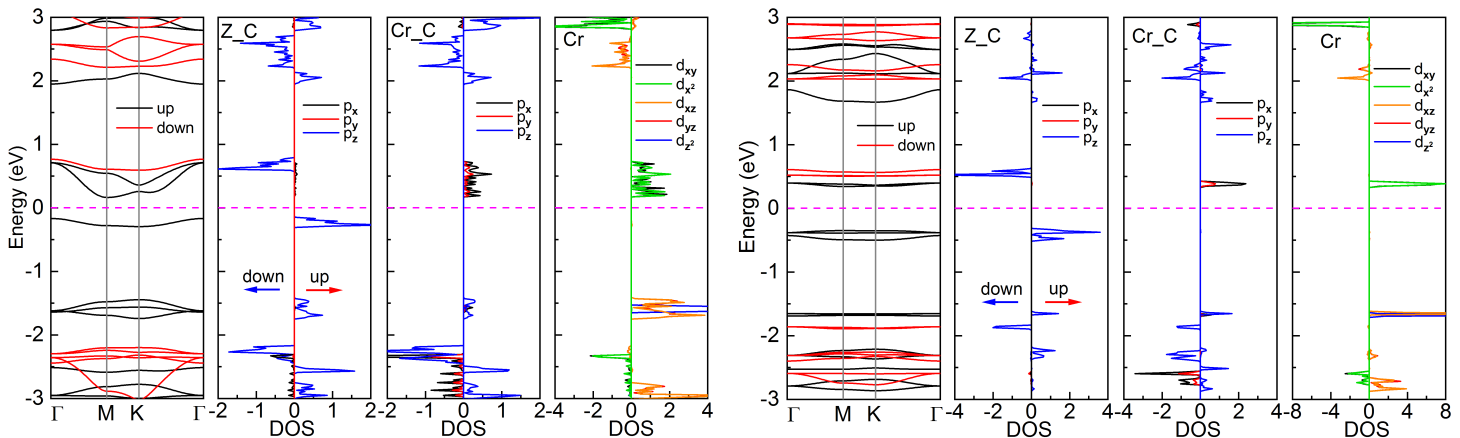

a

b
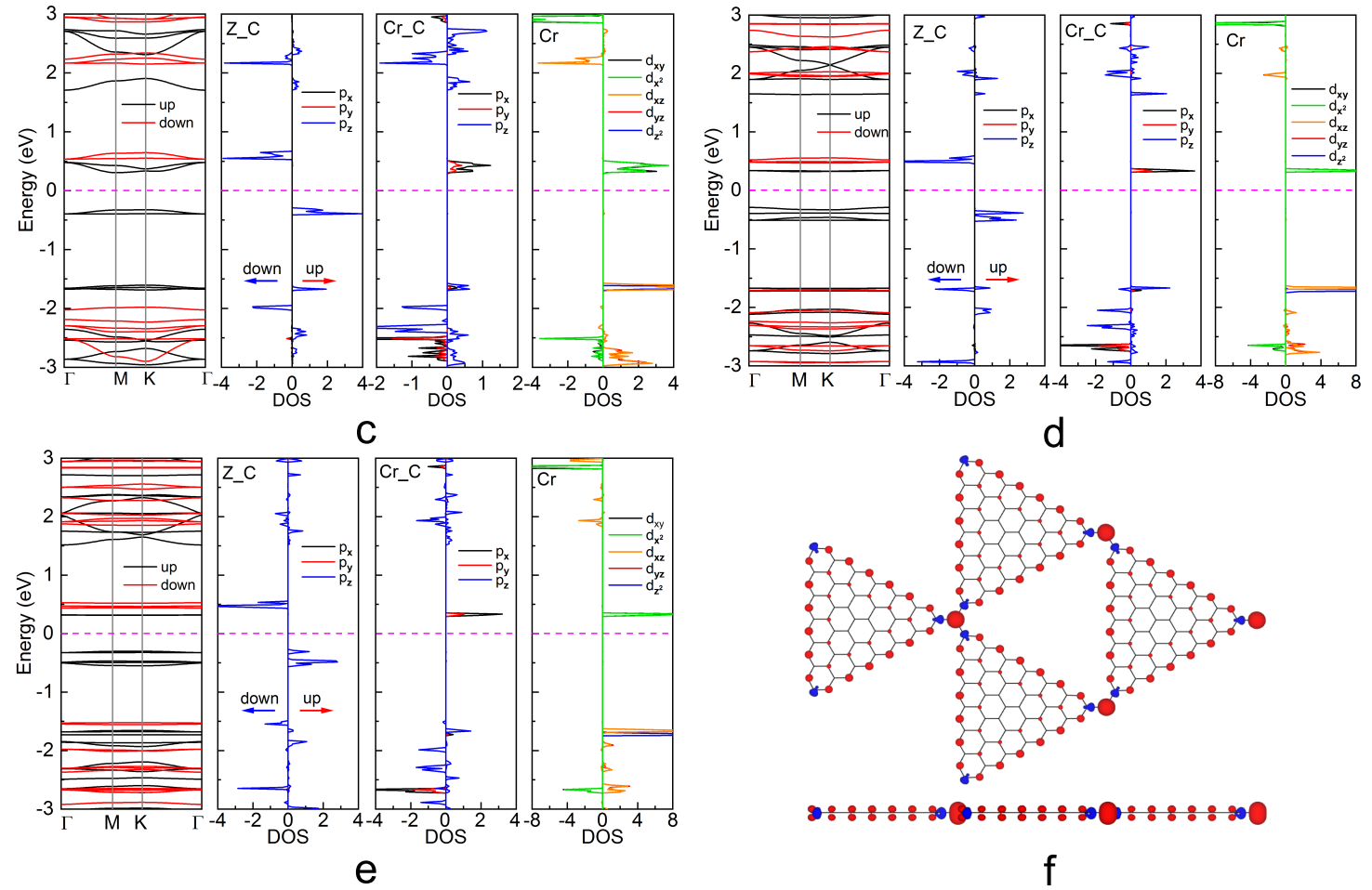

Figure S10 Band structures and projected density of states for the (a) TZGD_2_Cr, (b) TZGD_3_Cr, (c) TZGD_4_Cr, (d) TZGD_5_Cr, and (e) TZGD_6_Cr triangle lattices. The Fermi energy is set to zero. (f) Spin density of the TZGD_6_Cr triangle lattice with an isosurface value of $0.068 \mathrm{e} \cdot \AA^{-3}$. Red and blue regions represent the spin majority and spin minority. The hydrogen atoms are omitted. $\mathrm{Z}_{-} \mathrm{C}, \mathrm{Cr}_{-} \mathrm{C}$, and $\mathrm{Cr}$ denote the zigzag edge $\mathrm{C}$ atom, the interconnected $\mathrm{C}$ atom of TZGDs with $\mathrm{Cr}$, and the $\mathrm{Cr}$ atom. 

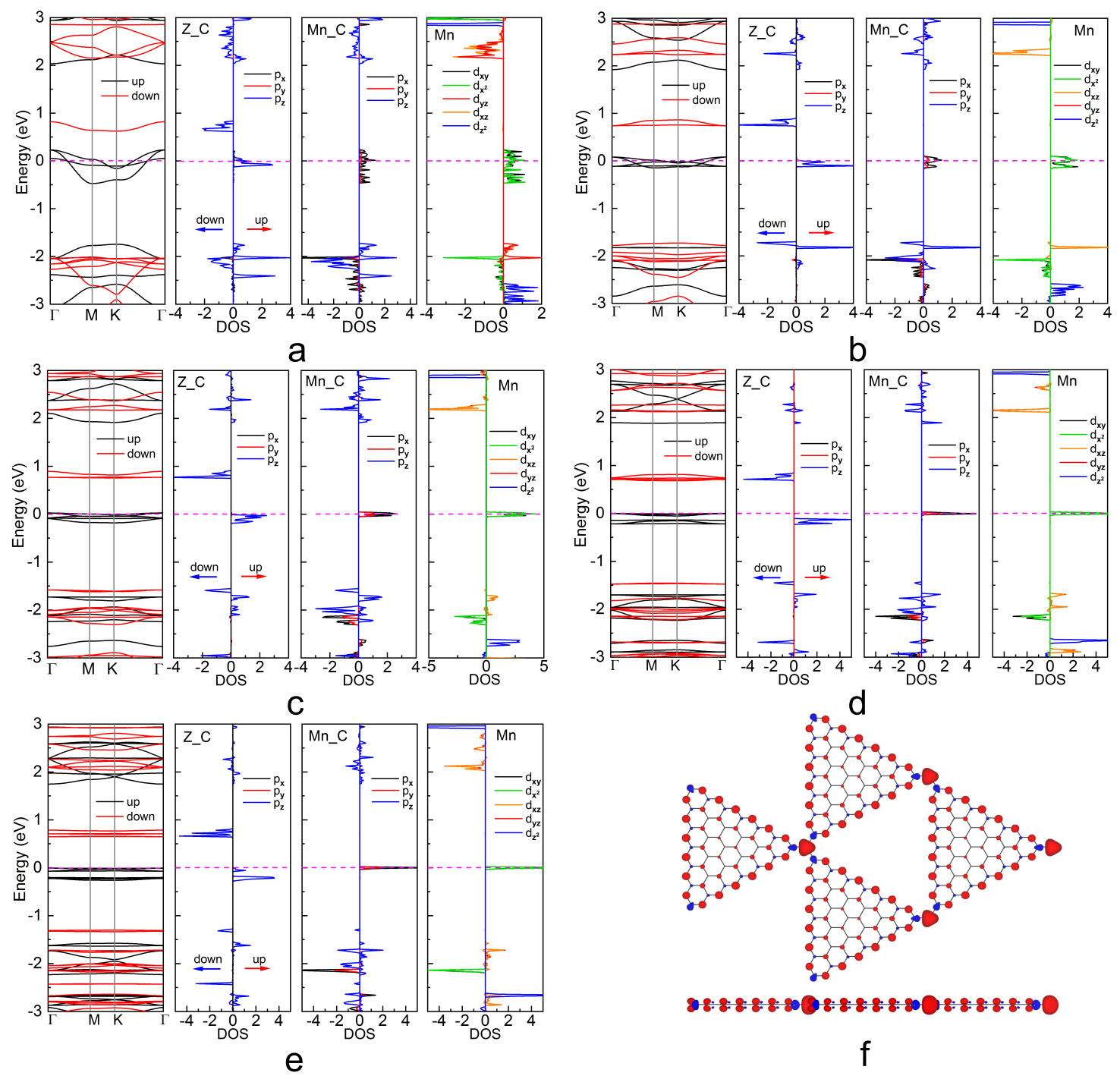

Figure S11 Band structures and projected density of states for the (a) TZGD_2_Mn, (b) TZGD_3_Mn, (c) TZGD_4_Mn, (d) TZGD_5_Mn, and (e) TZGD_6_Mn triangle lattices. The Fermi energy is set to zero. (f) Spin density of the TZGD_6_Mn triangle lattice with an isosurface value of $0.068 \mathrm{e} \cdot \AA^{-3}$. Red and blue regions represent the spin majority and spin minority. The hydrogen atoms are omitted. $\mathrm{Z}_{-} \mathrm{C}, \mathrm{Mn}_{-} \mathrm{C}$, and $\mathrm{Mn}$ denote the zigzag edge $\mathrm{C}$ atom, the interconnected $\mathrm{C}$ atom of TZGDs with $\mathrm{Mn}$, and the $\mathrm{Mn}$ atom. 

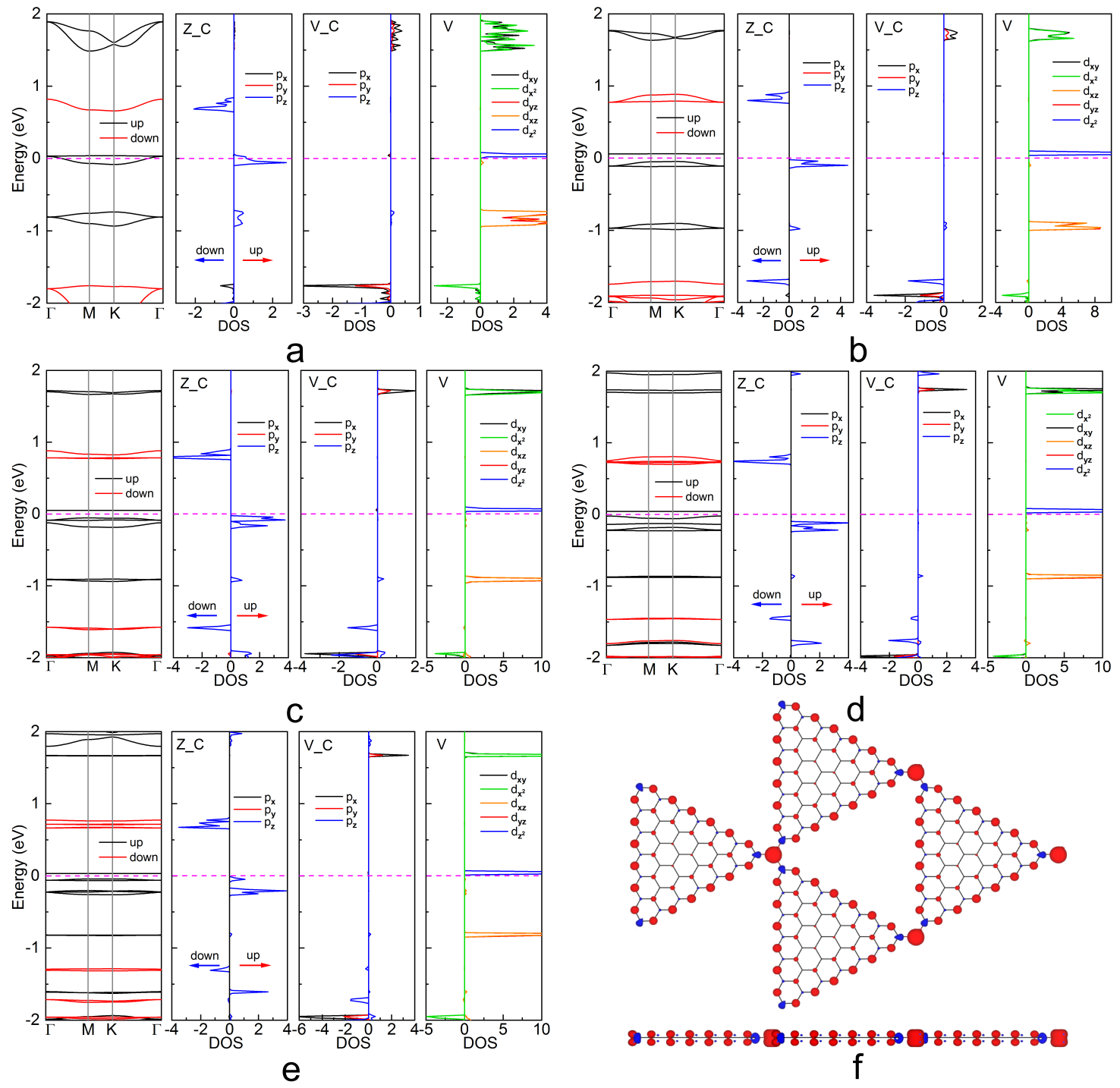

Figure S12 Band structures and projected density of states for the (a) TZGD_2_V, (b) TZGD_3_V, (c) TZGD_4_V, (d) TZGD_5_V, and (e) TZGD_6_V triangle lattices. The Fermi energy is set to zero. (f) Spin density of the TZGD_6_V triangle lattice with an isosurface value of $0.068 \mathrm{e} \cdot \AA^{-3}$. Red and blue regions represent the spin majority and spin minority. The hydrogen atoms are omitted. $\mathrm{Z}_{-} \mathrm{C}, \mathrm{V}_{-} \mathrm{C}$, and $\mathrm{V}$ denote the zigzag edge $\mathrm{C}$ atom, the interconnected $\mathrm{C}$ atom of TZGDs with $\mathrm{V}$, and the $\mathrm{V}$ atom. 

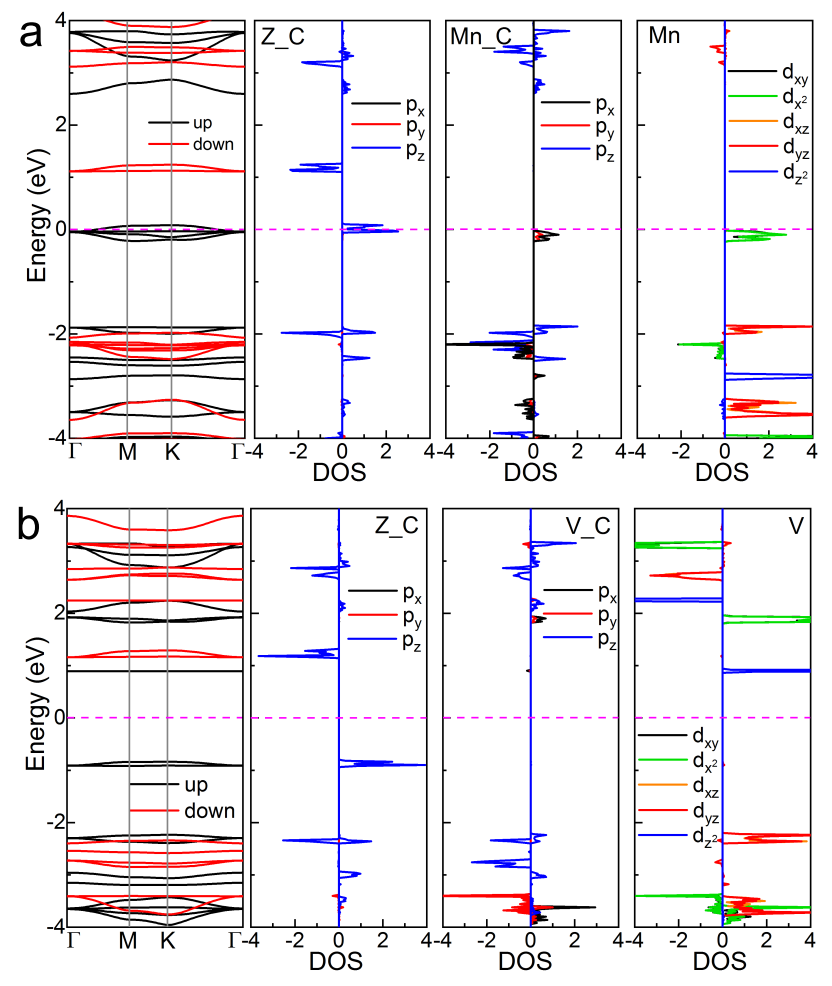

Figure S13 Band structures and projected density of states for (a) TZGD_3_Mn and (b) TZGD_3_V based on the HSE06 functional. Z_C, V/Mn_C, and V/Mn denote the zigzag edge C atom, the interconnected $\mathrm{C}$ atom of TZGDs with $\mathrm{V} / \mathrm{Mn}$, and the $\mathrm{V} / \mathrm{Mn}$ atom. 
Effect of the effective Hubbard U. Because of the strong Coulomb interaction in more localized d orbitals of the transition magnetic metal atoms, the effect of the effective Hubbard U parameter on the electronic structure of TZGD_3_L $(\mathrm{L}=\mathrm{V}, \mathrm{Cr}, \mathrm{Mn})$ is investigated (Table S3 and Figure S14). It can be seen that the U hardly changes the electronic structures and ground magnetic states of TZGD_3_L. Our calculated results confirm the PBE functional can give reliable electronic structures of TZGD_3_L.

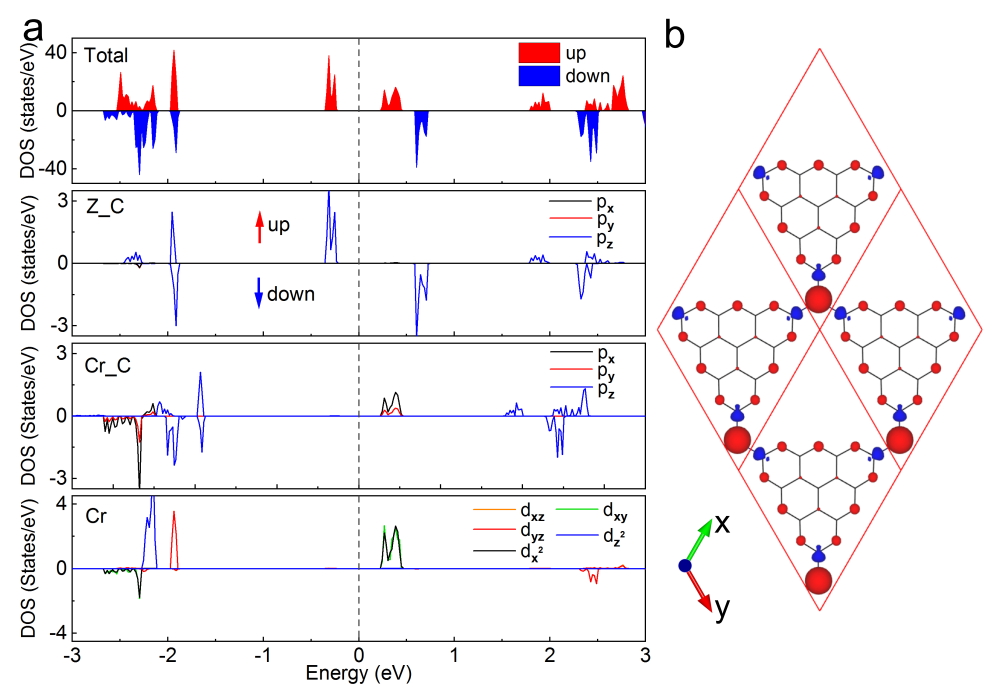

Figure S14 Projected density of states for (a) TZGD_3_Cr with $\mathrm{U}=3 \mathrm{eV}$. (b) Spin density of the TZGD_3_Cr triangle lattice with an isosurface value of $0.101 \mathrm{e} \cdot \AA^{-3}$ with $\mathrm{U}=3 \mathrm{eV} . \mathrm{Z}_{-} \mathrm{C}, \mathrm{Cr} \_\mathrm{C}$, and $\mathrm{Cr}$ denote the zigzag edge $\mathrm{C}$ atom, the interconnected $\mathrm{C}$ atom of TZGDs with $\mathrm{Cr}$, and the $\mathrm{Cr}$ atom. Red and blue regions represent the spin majority and spin minority. 
Table S3 Energy difference (eV) between different spin arrangements and FF for the triangle lattices based on TZGD_3 and transition magnetic metal atoms $(\mathrm{Cr}, \mathrm{Mn}, \mathrm{V})$ under different $\mathrm{U}$. The energy of FF is set to zero.

\begin{tabular}{cccccccc}
\hline \hline $\mathrm{M}$ & $\mathrm{U}$ & AA1 & AA2 & AF & FA & FF & FFA \\
\hline $\mathrm{V}$ & 1 & 0.400 & 0.297 & 0.309 & 0.295 & 0 & 0.615 \\
& 2 & 0.372 & 0.275 & 0.287 & 0.274 & 0 & 0.571 \\
& 3 & 0.348 & 0.259 & 0.270 & 0.258 & 0 & 0.539 \\
& 4 & 0.330 & 0.245 & 0.256 & 0.244 & 0 & 0.511 \\
& 5 & 0.314 & 0.232 & 0.244 & 0.233 & 0 & 0.488 \\
& 6 & 0.300 & 0.222 & 0.233 & 0.222 & 0 & 0.468 \\
& 7 & 0.288 & 0.213 & 0.224 & 0.214 & 0 & 0.451 \\
\hline $\mathrm{Cr}$ & 1 & 0.376 & 0.279 & 0.291 & 0.280 & 0 & 0.583 \\
& 2 & 0.361 & 0.268 & 0.279 & 0.270 & 0 & 0.562 \\
& 3 & 0.349 & 0.258 & 0.270 & 0.262 & 0 & 0.546 \\
& 4 & 0.340 & 0.251 & 0.262 & 0.255 & 0 & 0.532 \\
& 5 & 0.331 & 0.245 & 0.256 & 0.250 & 0 & 0.522 \\
& 6 & 0.326 & 0.240 & 0.251 & 0.246 & 0 & 0.514 \\
& 7 & 0.320 & 0.236 & 0.247 & 0.243 & 0 & 0.508 \\
\hline $\mathrm{Mn}$ & 1 & 0.379 & 0.297 & 0.284 & 0.213 & 0 & 0.404 \\
& 2 & 0.348 & 0.266 & 0.248 & 0.197 & 0 & 0.357 \\
& 3 & 0.320 & 0.241 & 0.216 & 0.184 & 0 & 0.318 \\
& 4 & 0.293 & 0.220 & 0.190 & 0.174 & 0 & 0.285 \\
& 5 & 0.268 & 0.202 & 0.167 & 0.164 & 0 & 0.256 \\
& 6 & 0.245 & 0.187 & 0.148 & 0.156 & 0 & 0.231 \\
& 7 & 0.224 & 0.173 & 0.131 & 0.148 & 0 & 0.210 \\
\hline \hline
\end{tabular}



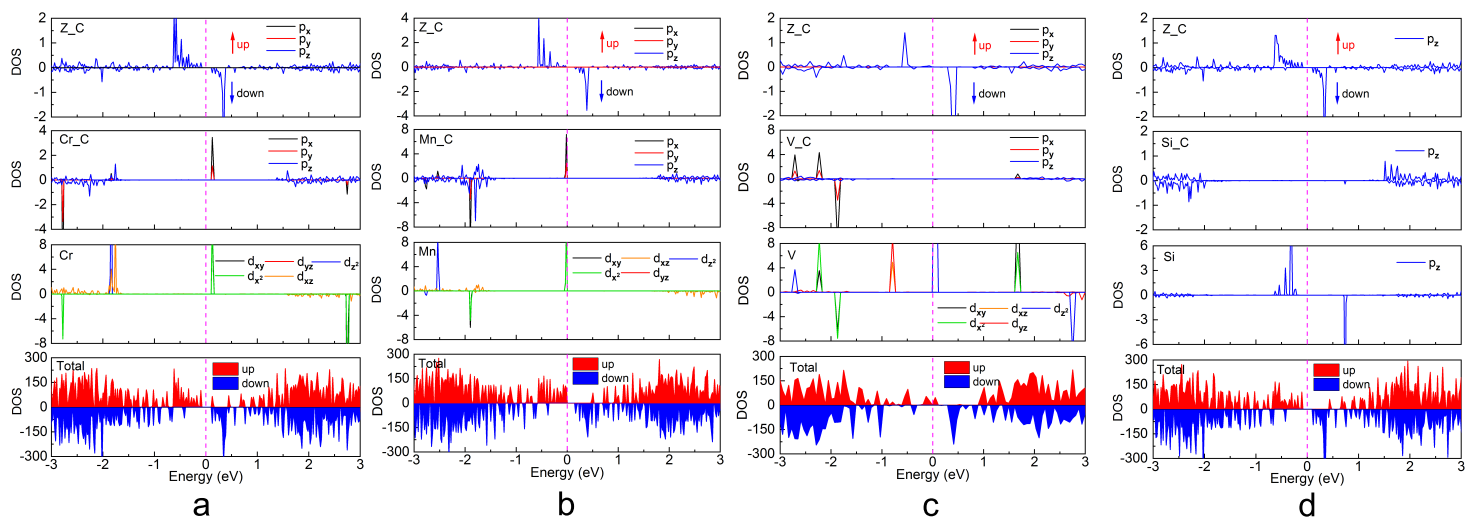

Figure S15 Projected density of states for the (a) TZGD_30_Cr, (b) TZGD_30_Mn, (c) TZGD_30_V, and (d) TZGD_30_Si triangle lattices. The Fermi energy is set to zero. 


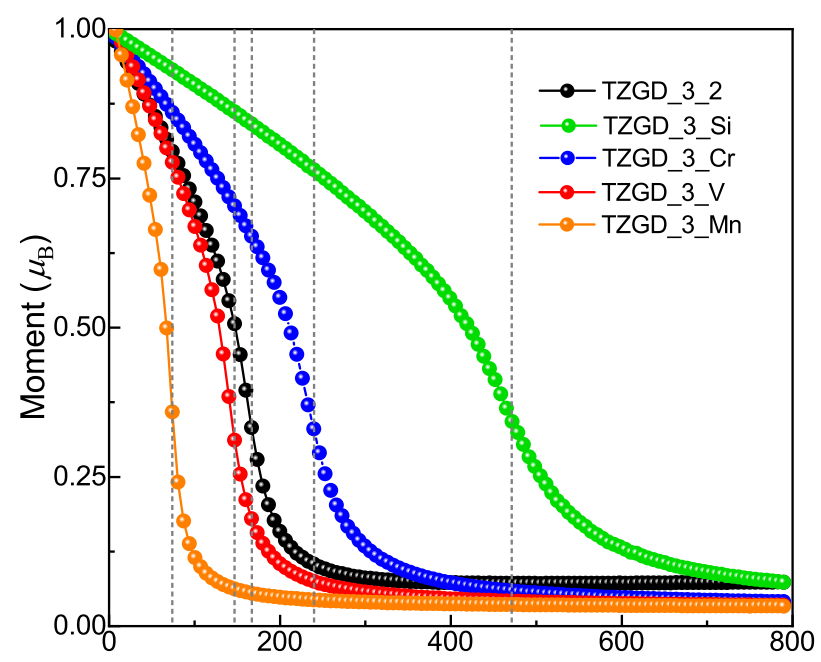

Figure S16 Magnetic moments with respect to temperature based on Monte Carlo simulations for the TZGD_3_L ( L = TZGD_2, Si, Cr, Mn, V) triangle lattices. Note that the total magnetic moments are set to 1 . 

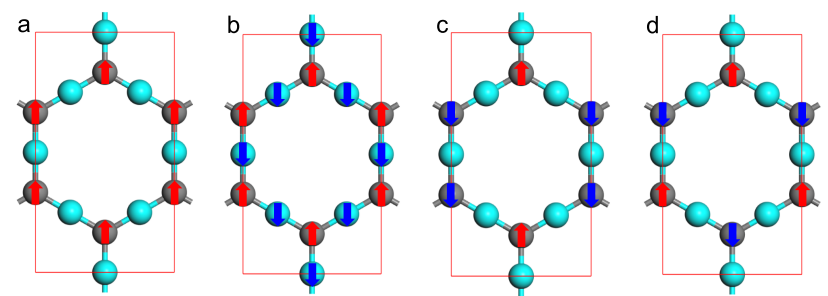

Figure S17 Four magnetic configurations in a $1 \times \sqrt{3} \times 1$ supercell for the kagome lattice. The blue and gray balls represent the linkages and TZGDs, respectively. (a) FF: TZGDs and linkages are in an FM arrangement, where the minority sublattice carbon atoms and linkages are in an AFM arrangement. (b) FFA: TZGDs and linkages are in an AFM arrangement. (c, d) AFM1 and AFM2: linkages are in an FM arrangement and TZGDs are in an AFM arrangement. Note: the magnetic configuration between TZGDs and linkages are defined by the majority sublattice carbon atoms and linkages. Red and blue arrows denote the spin polarization with different directions.

\section{D ferromagnets via linking TZGDs with atoms or molecules in kagome lat-}

tices. The $\mathrm{Cr}$ or Mn atoms are also engaged as linkages to connect TZGDs to form 2D TZGD_n_Cr and TZGD_n_Mn kagome lattices. Similar to the triangle lattices TZGD_n_L $(\mathrm{L}=\mathrm{Cr}, \mathrm{Mn})$, the calculated energy difference between magnetic configurations (Figure S17 and Table S4) confirms the FM ground state of these kagome lattices, where the TZGDs blocks and $\mathrm{Cr}$ or Mn atoms are in an FM alignment through the AFM coupling between the interconnected minority sublattice $\mathrm{C}$ atoms and linkages. Thus, 2D magnetic semiconductors and half-metallic ferromagnets are also realized in the TZGD_n_Cr and TZGD_n_Mn kagome lattices (Figure S18 and S19). The organic molecule, pentalene (P), also acts as a linkage to construct 2D TZGD_n_P kagome lattices with TZGDs blocks. Pentalene is an antiaromatic ring and possesses a localized closed-shell structure. ${ }^{32}$ It has been proved that pentalene is prone to spin polarization when connected with the magnetic center. ${ }^{33}$ TZGD_n_P kagome lattices take the FM arrangement, which is about $0.2 \mathrm{eV}$ lower than that of the AFM arrangement (Table S6). Through the direct exchange interaction, the interconnected $\mathrm{C}$ atoms of pentalene are antiparallel coupled with the apex $\mathrm{C}$ atoms of TZGDs. The electronic structures manifest the characteristic of a bipolar magnetic semiconductor (BMS) and the VBs and CBs near the Fermi level are dominated by the $\mathrm{p}_{z}$ orbitals of the edge $\mathrm{C}$ atoms in TZGDs and the $\mathrm{p}_{z}$ orbitals of pentalene (Figure S20), respectively. 
The total net magnetic moment of pentalene is zero and the magnetic moment of the entire system is twice that of single TZGDs blocks. Thus, by using pentalene, a series of COFs with the characteristic of BMSs with high-spin polarization can be also obtained.

Table S4 Energy difference (eV) between different spin arrangements and FF (Figure S17) for the TZGD_n_L $(\mathrm{n}=2,3,4,5,6 ; \mathrm{L}=\mathrm{Cr}, \mathrm{Mn})$ kagome lattices. The energy of FF is set to zero.

\begin{tabular}{cccccc}
\hline \hline M & TZGD_N & FF & AFM1 & AFM2 & AFM3 \\
\hline Cr & 2 & 0 & 0.276 & 0.136 & 0.136 \\
& 3 & 0 & 0.328 & 0.163 & 0.161 \\
& 4 & 0 & 0.332 & 0.165 & 0.163 \\
& 5 & 0 & 0.327 & 0.162 & 0.161 \\
& 6 & 0 & 0.326 & 0.162 & 0.160 \\
\hline Mn & 2 & 0 & FF & FF & FF \\
& 3 & 0 & 0.221 & 0.163 & 0.161 \\
& 4 & 0 & 0.274 & 0.137 & 0.136 \\
& 5 & 0 & 0.275 & 0.137 & 0.136 \\
& 6 & 0 & 0.290 & 0.144 & 0.143 \\
\hline \hline
\end{tabular}




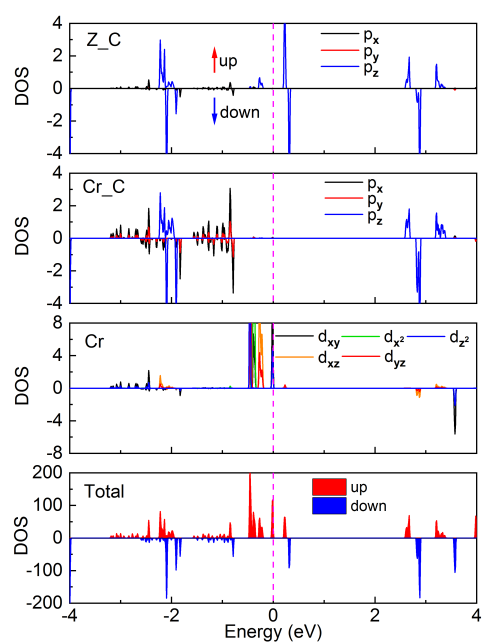

a

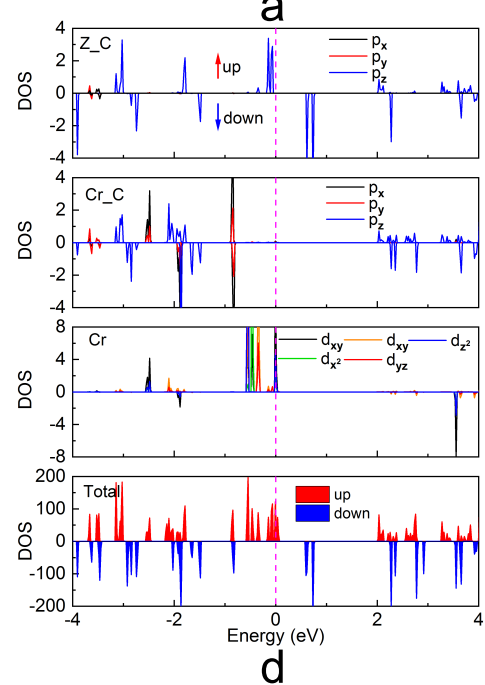

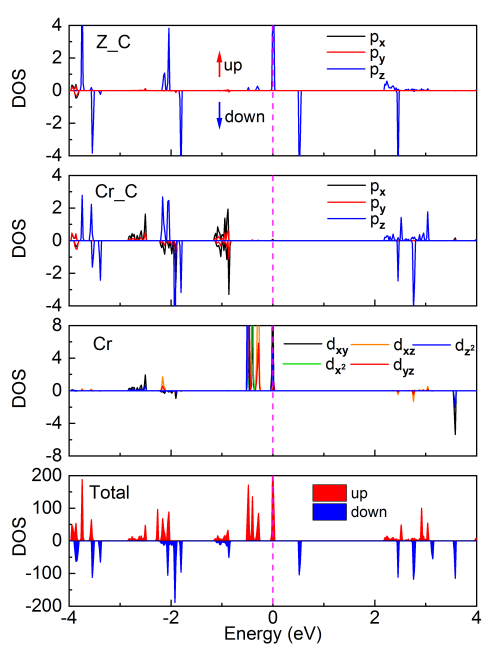

b

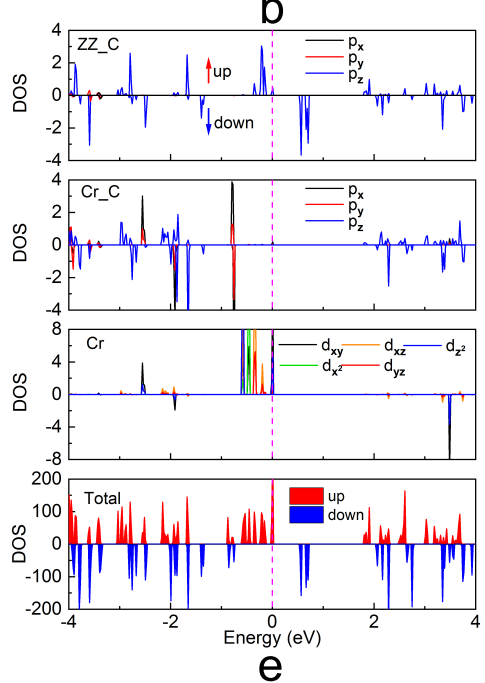

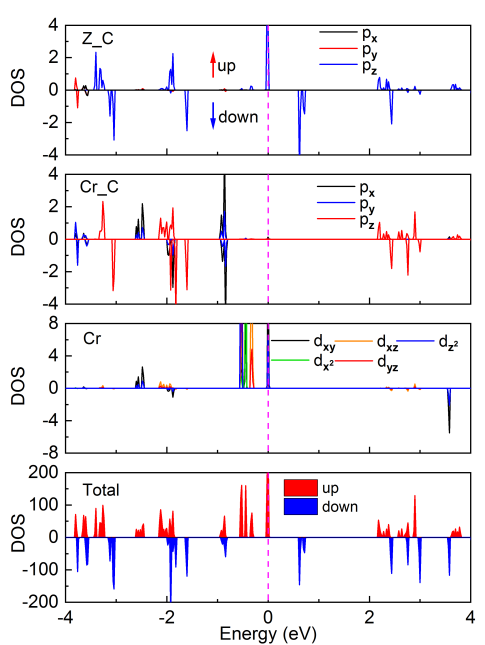

C

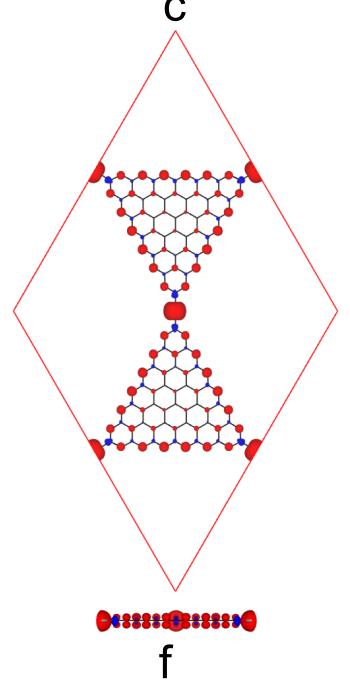

Figure S18 Projected density of states for the (a) TZGD_2_Cr, (b) TZGD_3_Cr, (c) TZGD_4_Cr, (d) TZGD_5_Cr, and (e) TZGD_6_Cr kagome lattices. The Fermi energy is set to zero. (f) Spin density of the TZGD_6_Cr kagome lattice with an isosurface value of $0.068 \mathrm{e} \cdot \AA^{-3}$. Red and blue regions represent the spin majority and spin minority. The hydrogen atoms are omitted. 

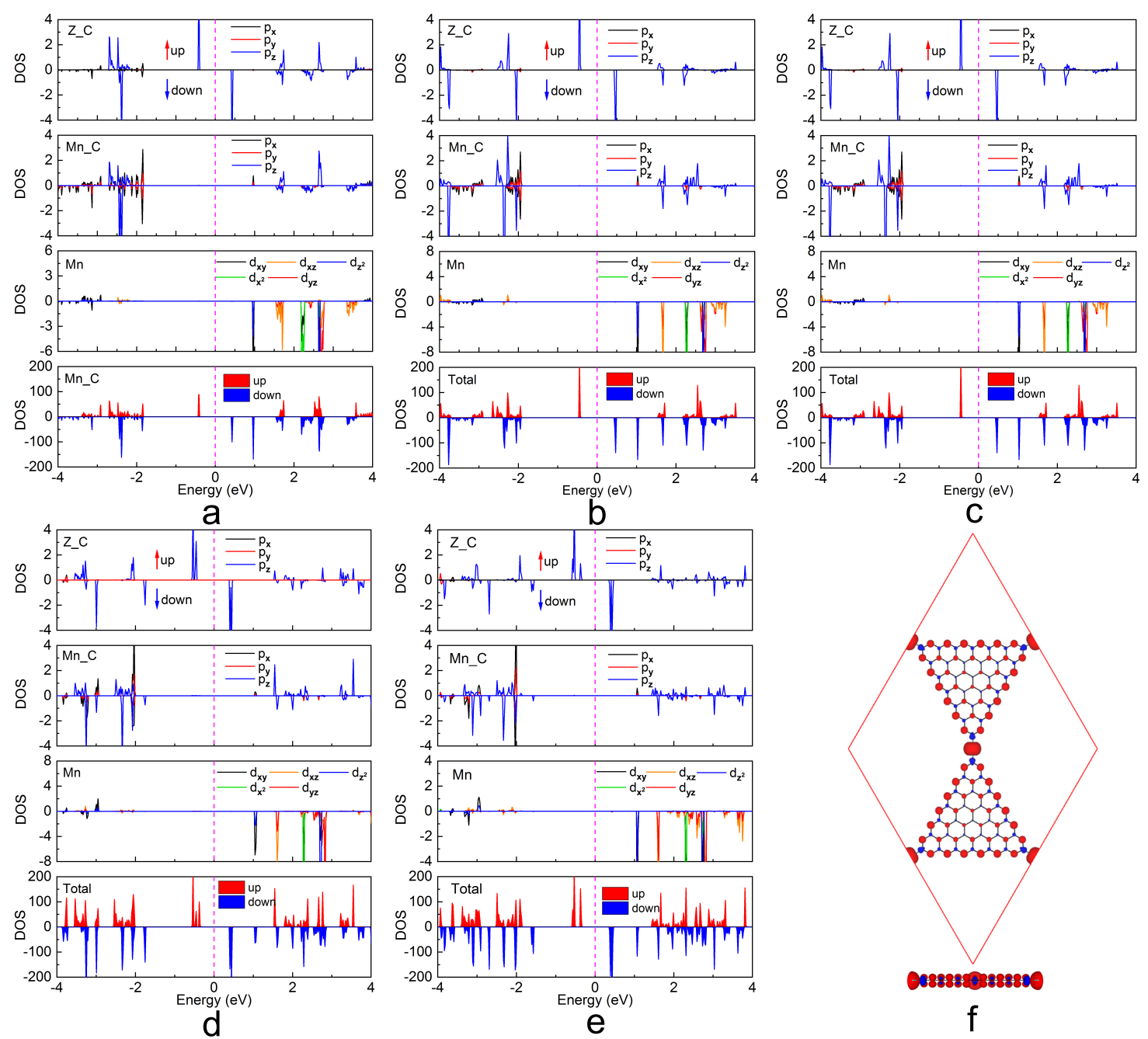

Figure S19 Projected density of states for the (a) TZGD_2_Mn, (b) TZGD_3_Mn, (c) TZGD_4_Mn, (d) TZGD_5_Mn, and (e) TZGD_6_Mn kagome lattices. The Fermi energy is set to zero. (f) Spin density of the TZGD_6_Mn kagome lattice with an isosurface value of 0.068 e. $\AA^{-3}$. Red and blue regions represent the spin majority and spin minority. The hydrogen atoms are omitted. 
Table S5 Energy difference $(\mathrm{eV})$ between different spin arrangements and FF for the TZGD_3_L $(\mathrm{L}=\mathrm{Cr}, \mathrm{Mn})$ kagome lattices under different $\mathrm{U}$. The energy of $\mathrm{FF}$ is set to zero.

\begin{tabular}{cccccc}
\hline \hline $\mathrm{M}$ & $\mathrm{U}$ & FF & AFM1 & AFM2 & AFM3 \\
\hline $\mathrm{Cr}$ & 1 & 0 & 0.198 & 0.099 & 0.099 \\
& 2 & 0 & 0.184 & 0.092 & 0.092 \\
& 3 & 0 & 0.173 & 0.086 & 0.086 \\
& 4 & 0 & 0.163 & 0.081 & 0.081 \\
& 5 & 0 & 0.155 & 0.077 & 0.081 \\
& 6 & 0 & 0.146 & 0.073 & 0.077 \\
& 7 & 0 & 0.138 & 0.069 & 0.073 \\
\hline $\mathrm{Mn}$ & 1 & 0 & 0.306 & 0.152 & 0.150 \\
& 2 & 0 & 0.286 & 0.142 & 0.140 \\
& 3 & 0 & 0.269 & 0.134 & 0.132 \\
& 4 & 0 & 0.254 & 0.126 & 0.124 \\
& 5 & 0 & 0.241 & 0.119 & 0.117 \\
& 6 & 0 & 0.229 & 0.123 & 0.112 \\
& 7 & 0 & 0.218 & 0.108 & 0.106 \\
\hline \hline
\end{tabular}

Table S6 Energy difference (eV) between FF and AFM for unit cell of the TZGD_n_P kagome lattices. FM and AFM correspond to the ferromagnetic and antiferromagnetic arrangements between TZGDs blocks, respectively. The energy of FM is set to zero.

\begin{tabular}{lccccc}
\hline \hline $\mathrm{N}$ & 2 & 3 & 4 & 5 & 6 \\
\hline FM & 0 & 0 & 0 & 0 & 0 \\
AFM & 0.155 & 0.195 & 0.194 & 0.194 & 0.199 \\
\hline \hline
\end{tabular}



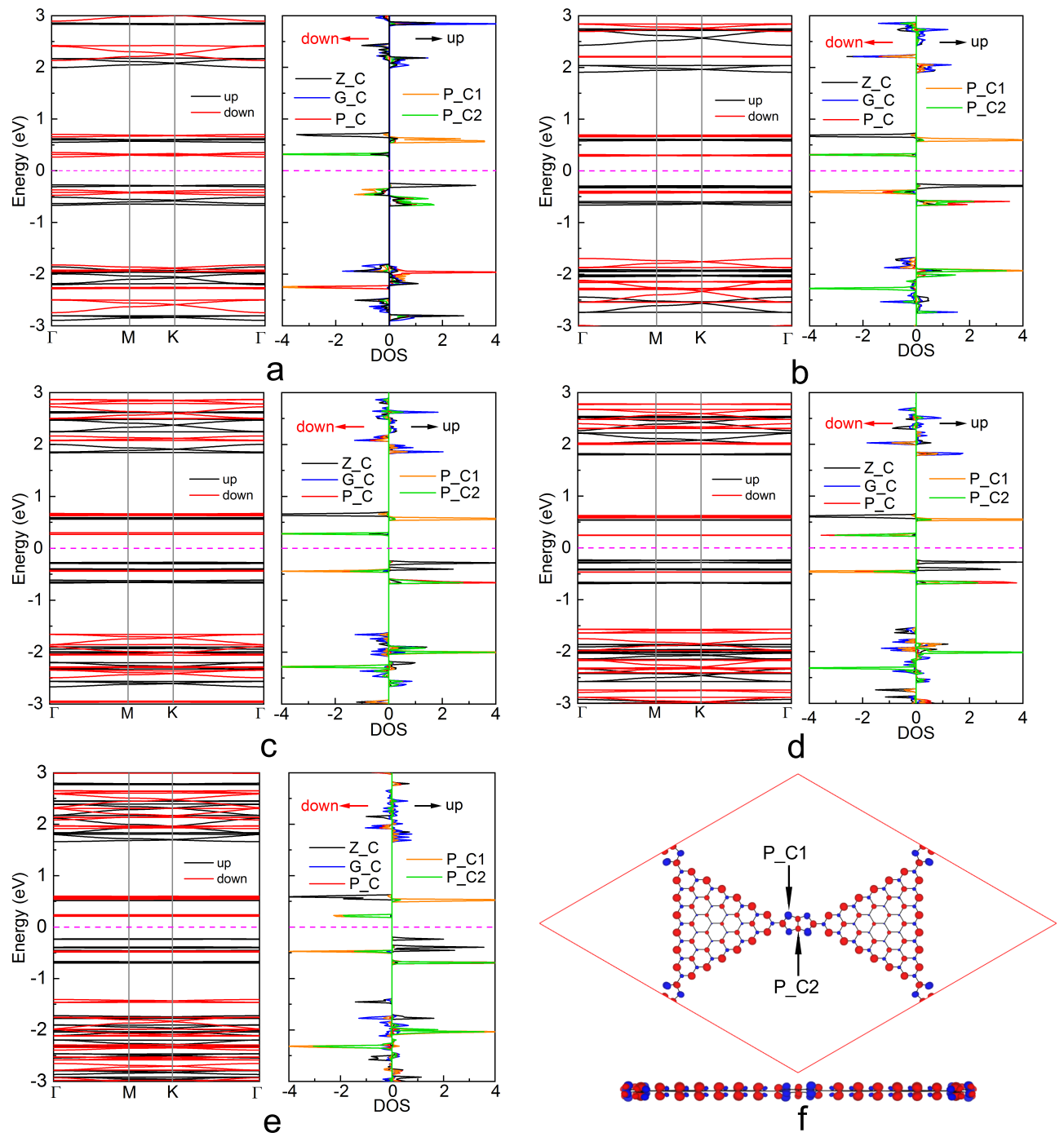

Figure S20 Band structures and projected density of states for the (a) TZGD_2_P, (b) TZGD_3_P, (c) TZGD_4_P, (d) TZGD_5_P, and (e) TZGD_6_P kagome lattices. The Fermi energy is set to zero. (f) Spin density of the TZGD_6_P kagome lattice with an isosurface value of $0.068 \mathrm{e} \cdot \AA^{-3}$. Red and blue regions represent the spin majority and spin minority. The hydrogen atoms are omitted. $\mathrm{Z}_{-} \mathrm{C}, \mathrm{G}_{-} \mathrm{C}, \mathrm{P}_{-} \mathrm{C}, \mathrm{P}_{-} \mathrm{C} 1$, and $\mathrm{P}_{-} \mathrm{C} 2$ denote the zigzag edge $\mathrm{C}$ atom, the interconnected $\mathrm{C}$ atom of TZGDs, the interconnected $\mathrm{C}$ atom of pentalene, and the $\mathrm{C}$ atoms of pentalene, as shown in (f). 
Ambient stability and experimental synthesis. To demonstrate the stability of these designed 2D COFs and MOFs based on TZGDs and various linkages, AIMD simulations are performed at 300 and $400 \mathrm{~K}$ by using an NVT ensemble lasting for 8.0 ps with a time step of 1.0 fs. For these investigated systems, the total energies converge quickly within 1.0 ps (Figure S21 and S22). Even the corresponding planar geometric structures fluctuate, these freestanding 2D lattices can still maintain their basic frameworks and there is no obvious bond break between the interconnected $\mathrm{C}$ atoms and linkages, which means these structures are stable at room temperature against thermal fluctuations. Furthermore, we have confirmed the stability of large-scale TZGDs (Figure S2 and S3). Since there is the almost same interaction strength between TZGDs with different sizes and linkages (L) with an almost same C-L bond length, it can be concluded that 2D triangle and kagome lattices constructed by large-scale TZGDs and linkages can remain stable at room temperature.

Usually, the $\mathrm{Au}(111)$ surface has been used as a substrate to synthesize TZGDs and carbon-based periodic lattices. ${ }^{23,34-38}$ The effect of substrate on the electronic structures of these 2D COFs and MOFs is also studied (Figure S23 and S24). For TZGD_5_2, the vertical distance between TZGD_5_2 and the $\mathrm{Au}(111)$ surface is calculated to be $3.12 \AA$, which is close to that between TZGD_5 monomer and the $\mathrm{Au}(111)$ surface $(3.27 \AA)$. The total magnetic moment of TZGD_5_2 is calculated to be $2.67 \mu_{\mathrm{B}}$, smaller than that of freestanding TZGD_5_2 $\left(3 \mu_{\mathrm{B}}\right)$, since about 0.4 electrons are injected into the substrate according to the Bader charge analysis. ${ }^{39}$ The PDOS manifests the electronic structure of TZGD_5_2 monolayer is hardly affected by the substrate, and the magnetic moments are still localized on the zigzag $\mathrm{C}$ atoms. In the case of the TZGD_5_Cr triangle lattice, the vertical distance between TZGD_5_Cr and the $\mathrm{Au}(111)$ surface is calculated to be $3.29 \AA$. There are about 0.09 electrons per unit cell transferred to the Au substrate, and TZGD_5_Cr can maintain its electronic structure basically. For the TZGD_3_Si triangle lattice, the calculated vertical distance between $\mathrm{Si}$ atoms and the $\mathrm{Au}(111)$ surface is $2.40 \AA$, and about 0.38 electrons per unit cell are injected into the Au substrate from TZGD_5_Si. Albeit with the almost 
annihilated magnetic moments of Si due to the transfer of charge, the magnetic moments of the zigzag $\mathrm{C}$ atoms are unchanged basically. Thus, the $\mathrm{Au}(111)$ surface is expected to be used as a substrate to synthesize TZGDs-based magnetic materials, TZGD_n_L.

When monolayer hexagonal boron nitride (hBN) is utilized to encapsulate the TZGD_3_2 monolayer, the vertical distance between hBN layers and TZGD_3_2 is $3.23 \AA$. The electronic structure is basically not affected by the $\mathrm{hBN}$ layer because of the weak vdW interaction (Figure S25). After 8 ps NVT simulations at room temperature, TZGD_3_2 layer becomes flatter compared with that of freestanding TZGD_3_2 (Figure S26). During the MD process, there is no clear structure destruction, which suggests that the hBN layer can stabilize TZGD_n_L. Additionally, graphene is a widely used in spintronics as a electrode. Here, we also check the influence of graphene $(\mathrm{Gr})$ on the electronic structures of these 2D ferromagnet, as shown in Figure S27. When the Gr monolayer is utilized to encapsulate the TZGD_n_L monolayer, the vertical distances between the Gr and TZGD_n_L layer are calculated to be $3.37,3.44$, and $3.55 \AA$ for the Gr/TZGD_3_2/Gr, Gr/TZGD_2_Cr/Gr, and Gr/TZGD_2_Si/Gr sandwich heterostructures, respectively. The PDOS of the TZGD_n_L layers in these heterostructures is similar to that of freestanding TZGD_n_L, which means TZGD_n_L layers still maintain their characteristics of BMS and FM semiconductor. In addition, the total spin moments $(2 \mathrm{~S})$ are calculated to be $0.84,7.14$ and $11.92 \mu_{\mathrm{B}}$, which are close to the values of 1,8 , and $12 \mu_{\mathrm{B}}$ for these freestanding TZGD_3_2, TZGD_2_Si, and TZGD_2_Cr supercell, respectively. Thus, it can be concluded that the corresponding electronic structures of TZGD_n_L are basically not affected by the Gr layer because of the weak vdW interaction. Last, we also check the thermostability of the Gr/TZGD_3_2/Gr. The total energy converges within 1.5 ps (Figure S28). After 4 ps NVT simulations at $300 \mathrm{~K}$, the TZGD_3_2 layer becomes flatter compared with that of freestanding TZGD_3_2, which is similar to the case of the hBN/TZGD_3_2/hBN. During the molecular dynamics process, there is also no clear structure destruction, which means that the Gr layer performing as a protective layer is also beneficial to stabilize these TZGD_n_L ferromagnets. 

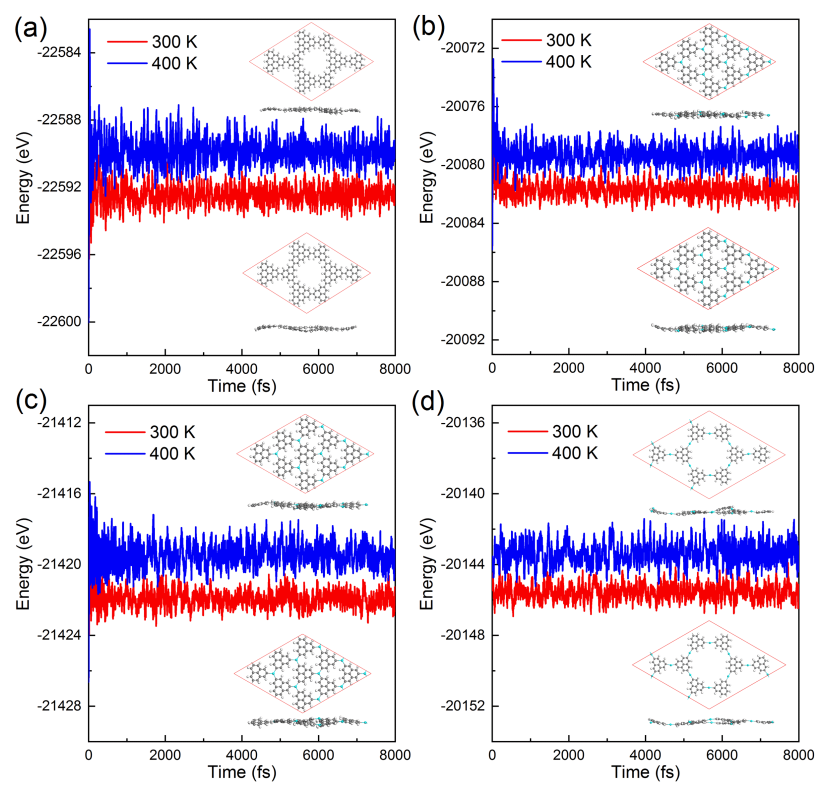

Figure S21 Energy fluctuations of the (a) TZGD_3_2, (b) TZGD_2_Si, (c) TZGD_2_Cr triangle lattices, and (d) TZGD_2_Cr kagome lattice as a function of time step by performing the AIMD simulation within 8 ps at 300 (red) and $400 \mathrm{~K}$ (blue). The inset graphs denote the top and side views of the optimized structure at the end of molecule dynamics process at 300 (bottom) and 400 K (top).

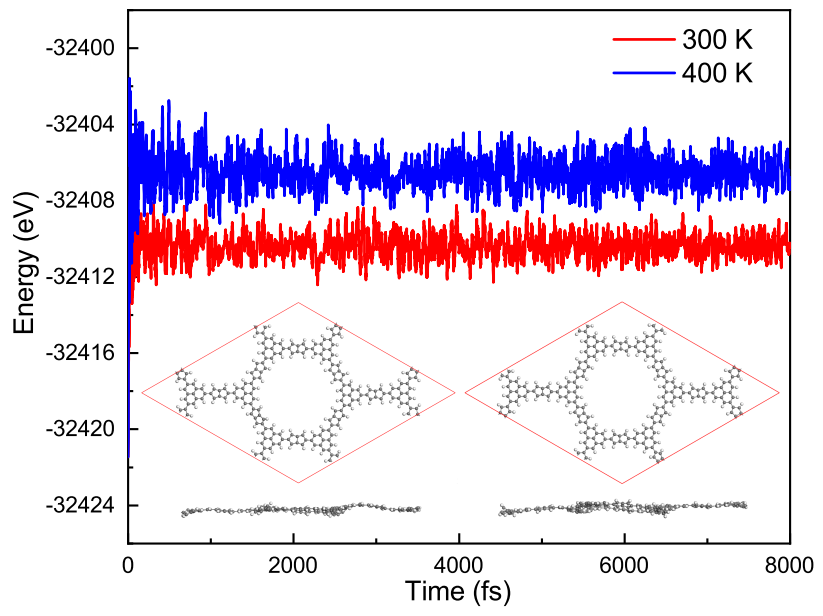

Figure S22 Energy fluctuations of TZGD_2_P as a function of time step by performing the AIMD simulation within 8 ps at 300 (red) and $400 \mathrm{~K}$ (blue). The inset graphs denote the top and side views of the optimized structures at the end of molecule dynamics process at 300 (left) and $400 \mathrm{~K}$ (right). 


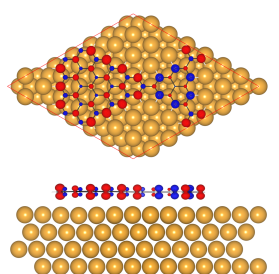

(a)
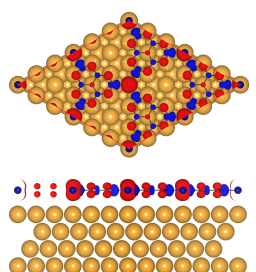

(b)
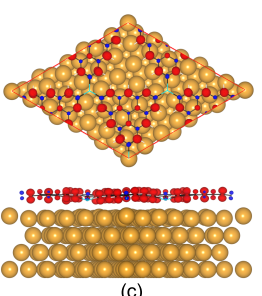

Figure S23 Spin density of the (a) TZGD_5_2, (b) TZGD_2_Cr, and (c) TZGD_5_Si triangle lattices on the $\mathrm{Au}(111)$ surface with an isosurface value of $0.038 \mathrm{e} / \AA^{3}$.
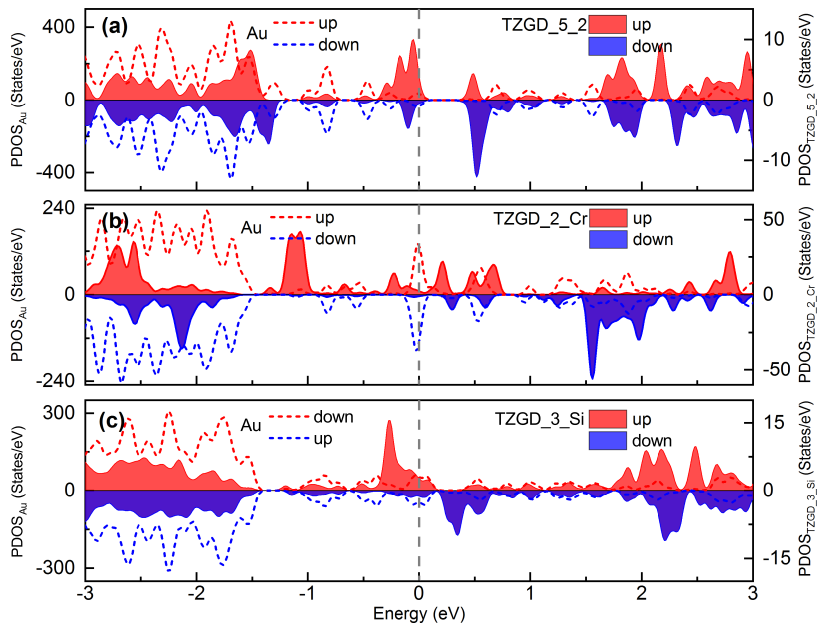

Figure S24 Projected density of states of the (a) TZGD_5_2, (b) TZGD_2_Cr, and (c) TZGD_3_Si triangle lattices on the $\mathrm{Au}(111)$ surface. The dash lines represent the projected density of states of $\mathrm{Au}$. The shaded area represents the projected density of states of TZGD_5_2, TZGD_2_Cr, and TZGD_3_Si. 

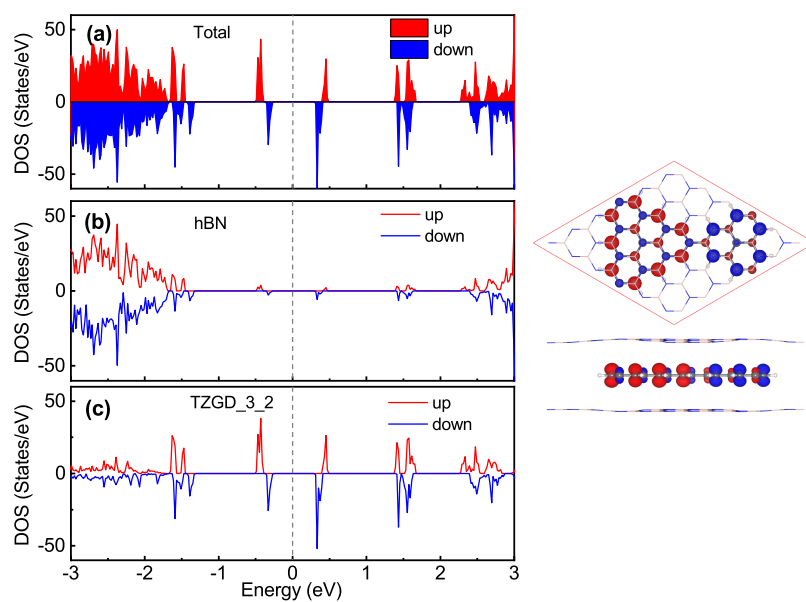

2008086

Figure S25 (a) Total density of states of the hBN/TZGD_3_2/hBN heterostructure and projected density of states of (b) the hBN layers and (c) TZGD_3_2. (d) Spin density for the hBN/TZGD_3_2/hBN heterostructure with an isosurface value of $0.038 \mathrm{e} / \AA^{3}$. The pink and blue line modes represent the $\mathrm{B}$ and $\mathrm{N}$ atoms. The grey and white balls denote the $\mathrm{C}$ and $\mathrm{H}$ atoms.

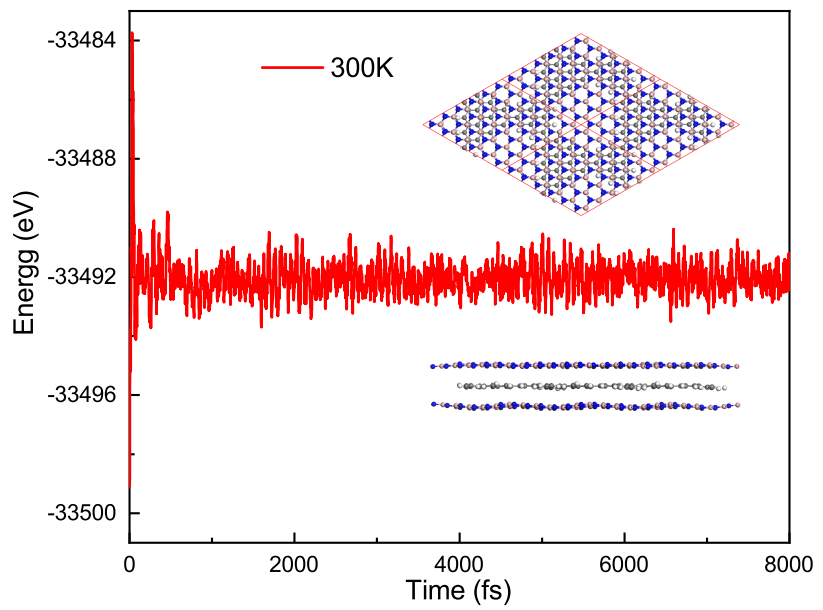

Figure S26 Energy fluctuations of the hBN/TZGD_3_2/hBN as a function of time step by performing the AIMD simulation within 8 ps at $300 \mathrm{~K}$. The insert graphs denote the optimized structure after 8 ps NVT simulation. 

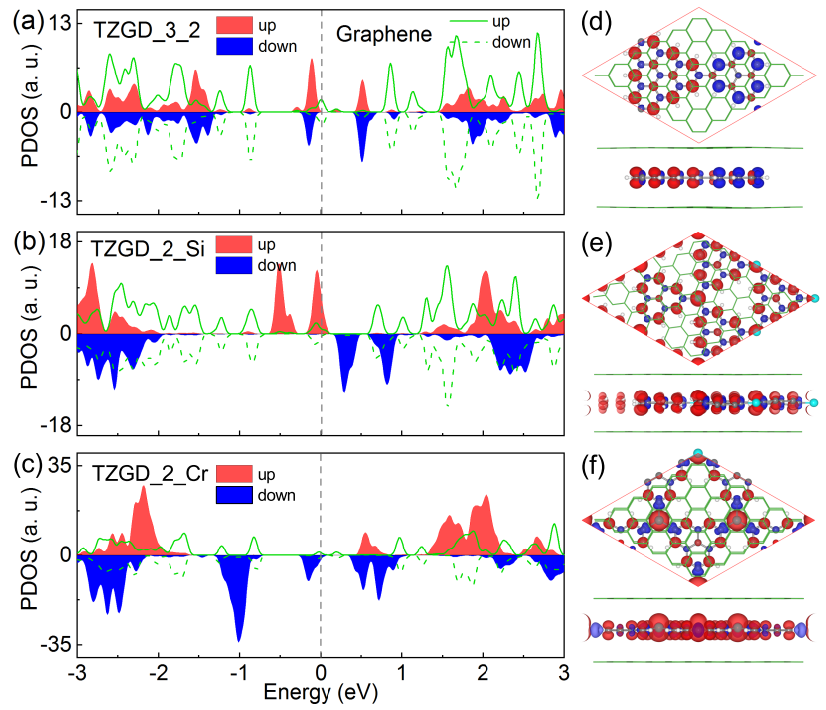

Figure S27 Projected density of states (PDOS) of the TZGD_n_L triangle lattices and graphene layer for the (a) Gr/TZGD_3_2/Gr, (b) Gr/TZGD_2_Si/Gr, and (c) Gr/TZGD_2_Cr/Gr sandwich heterostructures. The Fermi levels are set to zero. The red (blue) shadow areas and green solid (dash) lines denote the PDOS of TZGD_n_L and graphene layer, respectively. Spin density for the (d) Gr/TZGD_3_2/Gr, (e) Gr/TZGD_2_Si/Gr, and (f) Gr/TZGD_2_Cr/Gr with an isosurface value of $0.020,0.034$, and $0.027 \mathrm{e} / \AA^{3}$. The green honeycomb lattices represent the graphene layers. The grey, white, and cyan balls denote the $\mathrm{C}, \mathrm{H}$, and linkages ( $\mathrm{Si}, \mathrm{Cr}$ ), respectively. 


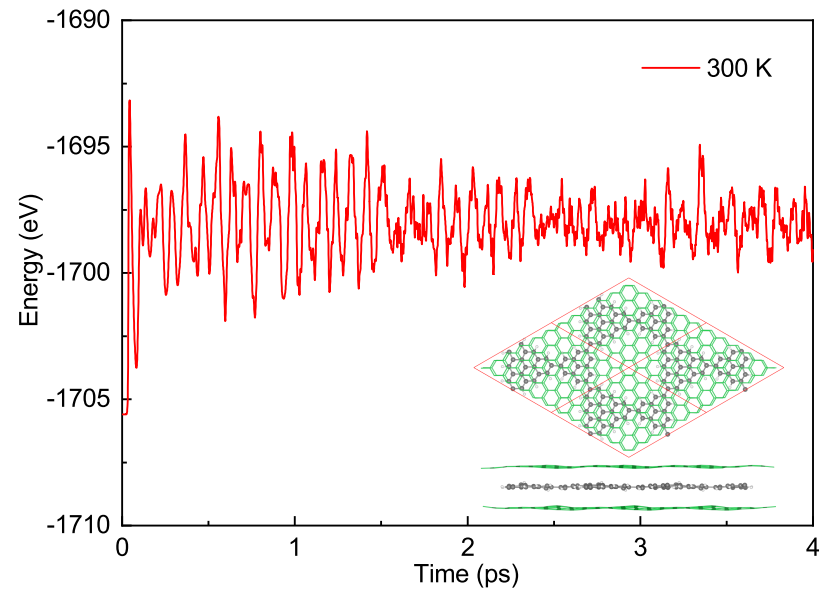

Figure S28 Energy fluctuation of the Gr/TZGD_3_2/Gr heterostructure as a function of time step by performing the AIMD simulation within 4 ps at $300 \mathrm{~K}$. The insert graphs denote the top and side views of the optimized structure after 4 ps NVT simulation. 
Magnetic anisotropy. Based on the $\mathrm{PBE}+\mathrm{SOC}$ (spin-orbit coupling) with including effective $\mathrm{U}=3 \mathrm{eV}$ for $\mathrm{Cr}, \mathrm{Mn}$ and $\mathrm{V}$, we calculate the magnetic anisotropy energy (MAE) for the TZGD_n_L triangle lattices. The MAE is defined as the $\triangle E=E_{=}-E_{\perp}$, where $E_{=}$ $\left(E_{\perp}\right)$ indicates the total energy for in-plane (out-of-plane) magnetic configuration. Through the calculation of the angular dependence of MAE with the magnetization in the $x y$ and $z x$ planes, as shown in Figure S29, we find the easy axis for TZGD_3_Cr and TZGD_3_Mn is out-of-plane, while the easy axis for TZGD_3_V is in-plane. Furthermore, the energy in the $z x$ plane is heavily dependent on the direction of the magnetization, whereas the energy is almost isotropic in the $x y$ plane. The MAEs for TZGD_3_Cr, TZGD_3_Mn, and TZGD_3_V are calculated to be 145,218 , and $109 \mu \mathrm{eV}$ per unit cell, respectively. Additionally, the band structures with the incorporation of spin-orbit coupling (SOC) are also investigated, as shown in Figure S30. It can be seen that SOC does not affect the semiconductor or metallic characteristics of these corresponding TZGD_3_L ( $\mathrm{L}=\mathrm{Cr}$, Mn, V) and the corresponding band structures are insensitive to SOC.

We re-evaluate the Curie temperature according to the generalized Heisenberg spin Hamiltonian with incorporation of the magnetic anisotropy, $H=-\sum_{i, j} J_{i, j} S_{i} S_{j}+\sum_{i} D_{i} S_{i z}^{2}$, where $J$ is the exchange-coupling parameter; $S$ is the spin magnetic moment; $D$ is the magnetic anisotropy parameter. ${ }^{40}$ Since the calculated magnetic anisotropy parameters $(D)$ is extremely small with the value of $0.06,0.03$, and $0.11 \mathrm{meV}$ for TZGD_3_Cr, TZGD_3_Mn, and TZGD_3_V, respectively, the Curie temperature remains basically unchanged with the incorporation of the magnetic anisotropy (Figure S31). 

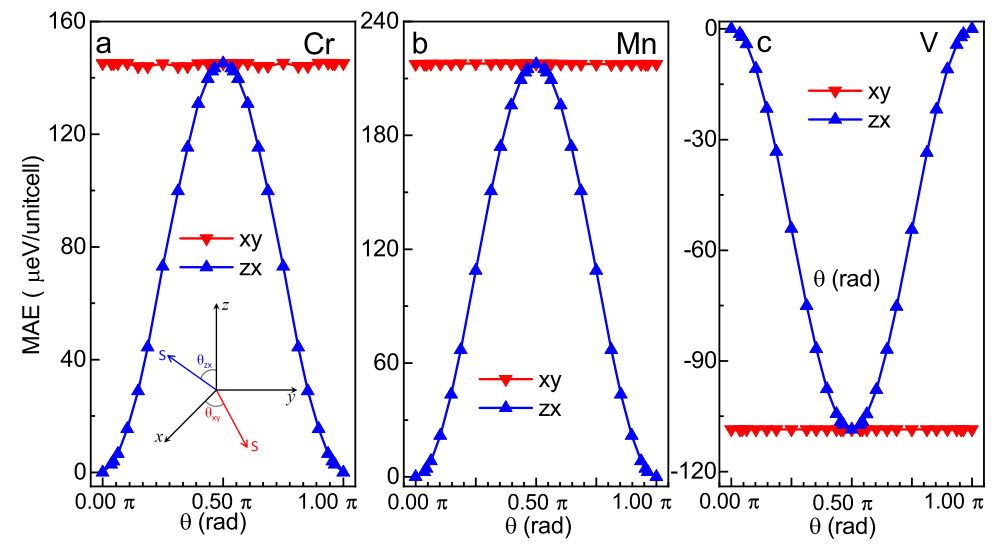

Figure S29 Angular dependence of magnetic anisotropy energy for the (a) TZGD_3_Cr, (b) TZGD_3_Mn, and (c) TZGD_3_V triangle lattices, with the magnetization in the $x y$ and $z x$ planes. The energy along the $\mathrm{z}$ axis is set to zero. The inset graph in (a) denote the magnetization vector $S$ with a rotation angle $\theta_{x y}$ and $\theta_{z x}$ on the $x y$ and $z x$ plane.
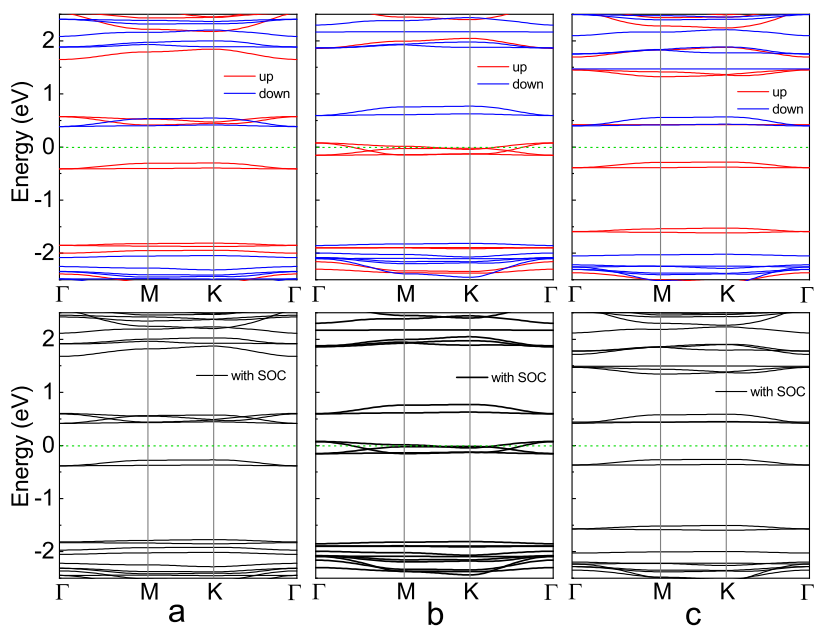

Figure S30 Band structures of the (a) TZGD_3_Cr, (b) TZGD_3_Mn, and (c) TZGD_3_V triangle lattices without the incorporation of spin-orbit coupling (SOC) (top) and with SOC (bottom). 


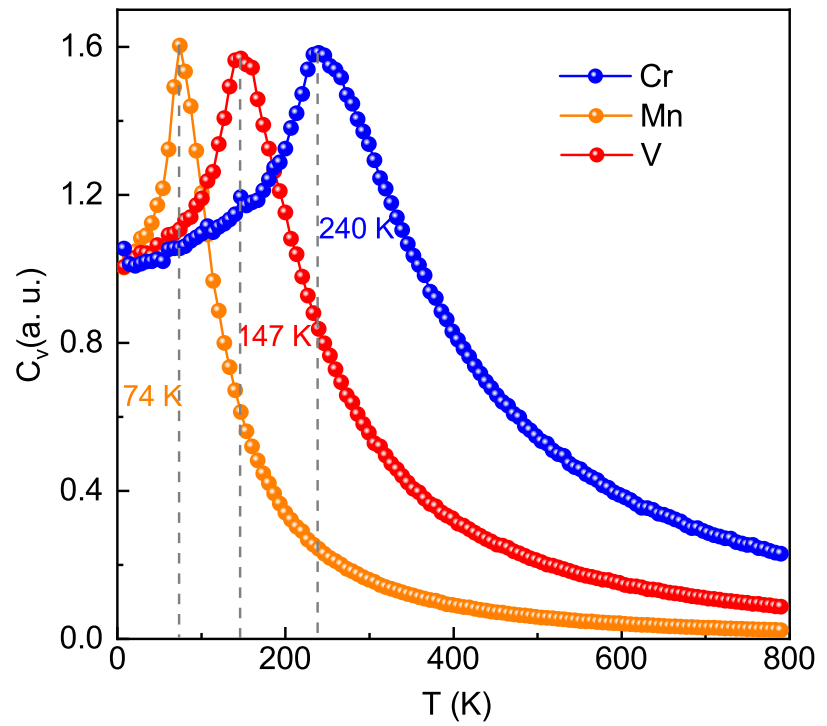

Figure S31 Specific heat $\left(\mathrm{C}_{V}\right)$ with respect to temperature from Monte Carlo simulations with the incorporation of spin-orbit coupling for the TZGD_3_L $(\mathrm{L}=\mathrm{Cr}, \mathrm{Mn}, \mathrm{V})$ triangle lattices. 


\section{References}

(1) Soler, J. M.; Artacho, E.; Gale, J. D.; García, A.; Junquera, J.; Ordejón, P.; Sánchez-Portal, D. The SIESTA method for ab initio order-N materials simulation. J. Phys. Condens. Matter 2002, 14, 27452779 .

(2) Perdew, J. P.; Burke, K.; Ernzerhof, M. Generalized gradient approximation made simple. Phys. Rev. Lett. 1996, 77, 3865-3868.

(3) Junquera, J.; Paz, Ó.; Sánchez-Portal, D.; Artacho, E. Numerical atomic orbitals for linear-scaling calculations. Phys. Rev. B 2001, 64, 235111.

(4) Heyd, J.; Scuseria, G. E.; Ernzerhof, M. Hybrid functionals based on a screened Coulomb potential. $J$. Chem. Phys. 2003, 118, 8207-8215.

(5) Heyd, J.; Scuseria, G. E.; Ernzerhof, M. Erratum: "Hybrid functionals based on a screened Coulomb potential" [J. Chem. Phys. 118, 8207 (2003)]. J. Chem. Phys. 2006, 124, 219906.

(6) Qin, X.; Shang, H.; Xiang, H.; Li, Z.; Yang, J. HONPAS: A linear scaling open-source solution for large system simulations. Int. J. Quantum. Chem. 2015, 115, 647-655.

(7) Shang, H.; Li, Z.; Yang, J. Implementation of exact exchange with numerical atomic orbitals. J. Phys. Chem. A 2010, 114, 1039-1043.

(8) Shang, H.; Li, Z.; Yang, J. Implementation of screened hybrid density functional for periodic systems with numerical atomic orbitals: basis function fitting and integral screening. J. Chem. Phys. 2011, $135,034110$.

(9) Qin, X.; Liu, J.; Hu, W.; Yang, J. Interpolative separable density fitting decomposition for accelerating Hartree-Fock exchange calculations within numerical atomic orbitals. J. Phys. Chem. A 2020, 124, $5664-5674$.

(10) Hu, W.; Huang, Y.; Qin, X.; Lin, L.; Kan, E.; Li, X.; Yang, C.; Yang, J. Room-temperature magnetism and tunable energy gaps in edge-passivated zigzag graphene quantum dots. npj 2D Mater. Appl. 2019, 3, 17.

(11) Hu, W.; Lin, L.; Yang, C.; Yang, J. Electronic structure and aromaticity of large-scale hexagonal graphene nanoflakes. J. Chem. Phys. 2014, 141, 214704. 
(12) Kresse, G.; Furthmüller, J. Efficiency of ab-initio total energy calculations for metals and semiconductors using a plane-wave basis set. Comput. Mater. Sci. 1996, 6, 15-50.

(13) Kresse, G.; Furthmüller, J. Efficient iterative schemes for ab initio total-energy calculations using a plane-wave basis set. Phys. Rev. B 1996, 54, 11169-11186.

(14) Grimme, S. Semiempirical GGA-type density functional constructed with a long-range dispersion correction. J. Comput. Chem. 2006, 27, 1787-1799.

(15) Bieri, M.; Blankenburg, S.; Kivala, M.; Pignedoli, C. A.; Ruffieux, P.; Müllen, K.; Fasel, R. Surfacesupported 2D heterotriangulene polymers. Chem. Commun. 2011, 47, 10239-10241.

(16) Nørskov, J. K.; Studt, F.; Abild-Pedersen, F.; Bligaard, T. Fundamental concepts in heterogeneous catalysis; John Wiley \& Sons, Inc: Hoboken, NJ, 2014.

(17) Wang, W. L.; Meng, S.; Kaxiras, E. Graphene nanoflakes with large spin. Nano Lett. 2008, 8, 241-245.

(18) Mishra, S.; Beyer, D.; Eimre, K.; Ortiz, R.; Fernández-Rossier, J.; Berger, R.; Gröning, O.; Pignedoli, C. A.; Fasel, R.; Feng, X., et al. Collective all-carbon magnetism in triangulene dimers. Angew. Chem., Int. Ed. 2020, 59, 12041-12047.

(19) Ortiz, R.; Boto, R. A.; García-Martínez, N.; Sancho-García, J. C.; Melle-Franco, M.; FernándezRossier, J. Exchange rules for diradical $\pi$-conjugated hydrocarbons. Nano Lett. 2019, 19, 5991-5997.

(20) Lieb, E.; Mattis, D. Ordering energy levels of interacting spin systems. J. Math. Phys. 1962, 3, 749-751.

(21) Lieb, E. H. Two theorems on the Hubbard model. Phys. Rev. Lett. 1989, 62, 1201-1204.

(22) Deng, Q.; Chai, J.-D. Electronic properties of triangle-shaped graphene nanoflakes from TAO-DFT. ACS Omega 2019, 4, 14202-14210.

(23) Su, J.; Telychko, M.; Hu, P.; Macam, G.; Mutombo, P.; Zhang, H.; Bao, Y.; Cheng, F.; Huang, Z.-Q.; Qiu, Z., et al. Atomically precise bottom-up synthesis of $\pi$-extended [5]triangulene. Sci. Adv. 2019, 5, eaav7717.

(24) Zhou, Y.; Liu, F. Realization of an antiferromagnetic superatomic graphene: Dirac Mott insulator and circular dichroism Hall effect. Nano Lett. 2021, 21, 230-235.

(25) Sethi, G.; Zhou, Y.; Zhu, L.; Yang, L.; Liu, F. Flat-band-enabled triplet excitonic insulator in a diatomic Kagome lattice. Phys. Rev. Lett. 2021, 126, 196403. 
(26) Melle-Franco, M. When 1+ 1 is odd. Nat. Nanotechnol. 2017, 12, 292-293.

(27) Zheng, Y.; Li, C.; Zhao, Y.; Beyer, D.; Wang, G.; Xu, C.; Yue, X.; Chen, Y.; Guan, D.-D.; Li, Y.-Y., et al. Engineering of magnetic coupling in nanographene. Phys. Rev. Lett. 2020, 124, 147206.

(28) Wang, Y.; Guo, Y.; Wang, Z.; Fu, L.; Zhang, Y.; Xu, Y.; Yuan, S.; Pan, H.; Du, Y.; Wang, J., et al. Realization of strong room-temperature ferromagnetism in atomically thin $2 \mathrm{D}$ carbon nitride sheets by thermal annealing. ACS Nano 2021, 15, 12069-12076.

(29) Mishra, S.; Yao, X.; Chen, Q.; Eimre, K.; Gröning, O.; Ortiz, R.; Di Giovannantonio, M.; SanchoGarcía, J. C.; Fernández-Rossier, J.; Pignedoli, C. A., et al. Large magnetic exchange coupling in rhombus-shaped nanographenes with zigzag periphery. Nat. Chem. 2021, 13, 581-586.

(30) Pinkard, A.; Champsaur, A. M.; Roy, X. Molecular clusters: nanoscale building blocks for solid-state materials. Acc. Chem. Res. 2018, 51, 919-929.

(31) Silva, A.; Pires, M.; Freire, V.; Albuquerque, E.; Azevedo, D.; Caetano, E. Graphene nanoflakes: thermal stability, infrared signatures, and potential applications in the field of spintronics and optical nanodevices. J. Phys. Chem. C 2010, 114, 17472-17485.

(32) Baird, N.; West, R. Applications of quantum chemistry. II. Ground and excited states of pentalene. $J$. Am. Chem. Soc. 1971, 93, 3072-3073.

(33) Li, X.; Yang, J. Realizing two-dimensional magnetic semiconductors with enhanced Curie temperature by antiaromatic ring based organometallic frameworks. J. Am. Chem. Soc. 2019, 141, 109-112.

(34) Mishra, S.; Beyer, D.; Eimre, K.; Liu, J.; Berger, R.; Gröning, O.; Pignedoli, C. A.; Müllen, K.; Fasel, R.; Feng, X.; Ruffieux, P. Synthesis and characterization of $\pi$-extended triangulene. J. Am. Chem. Soc. 2019, 141, 10621-10625.

(35) Pavliček, N.; Mistry, A.; Majzik, Z.; Moll, N.; Meyer, G.; Fox, D. J.; Gross, L. Synthesis and characterization of triangulene. Nat. Nanotechnol. 2017, 12, 308-311.

(36) Su, J.; Fan, W.; Mutombo, P.; Peng, X.; Song, S.; Ondráček, M.; Golub, P.; Brabec, J.; Veis, L.; Telychko, M., et al. On-Surface synthesis and characterization of [7]triangulene quantum ring. Nano Lett. 2021, 21, 861-867. 
(37) Mishra, S.; Beyer, D.; Berger, R.; Liu, J.; Gröning, O.; Urgel, J. I.; Müllen, K.; Ruffieux, P.; Feng, X.; Fasel, R. Topological defect-induced magnetism in a nanographene. J. Am. Chem. Soc. 2020, 142, $1147-1152$.

(38) Galeotti, G.; De Marchi, F.; Hamzehpoor, E.; MacLean, O.; Rajeswara Rao, M.; Chen, Y.; Besteiro, L. V.; Dettmann, D.; Ferrari, L.; Frezza, F., et al. Synthesis of mesoscale ordered twodimensional $\pi$-conjugated polymers with semiconducting properties. Nat. Mater. 2020, 19, 874-880.

(39) Tang, W.; Sanville, E.; Henkelman, G. A grid-based Bader analysis algorithm without lattice bias. J. Phys. Condens. Matter 2009, 21, 084204.

(40) Xiang, H.; Wei, S.-H.; Whangbo, M.-H. Origin of the structural and magnetic anomalies of the layered compound $\mathrm{SrFeO}_{2}$ : a density functional investigation. Phys. Rev. Lett. 2008, 100, 167207. 